\title{
An amygdala circuit that suppresses social engagement
}

3 Jeong-Tae Kwon ${ }^{1,2}$, Changhyeon Ryu ${ }^{1,2 \dagger}$, Hyeseung Lee ${ }^{1,2,4 \dagger}$, Alec Sheffield ${ }^{1,2}$, Jingxuan Fan ${ }^{2}$,

4 Daniel H. Cho ${ }^{2}$, Shivani Bigler ${ }^{2}$, Heather A. Sullivan ${ }^{3}$, Han Kyung Choe ${ }^{2}$, Ian R. Wickersham ${ }^{3}$,

5 Myriam Heiman ${ }^{1,2,4}$ \& Gloria B. Choi ${ }^{1,2, *}$

6

7 'Picower Institute for Learning and Memory, Massachusetts Institute of Technology, Cambridge, MA 02139, USA.

$8{ }^{2}$ Department of Brain and Cognitive Sciences, Massachusetts Institute of Technology, Cambridge, MA 02139, USA.

$9{ }^{3}$ McGovern Institute for Brain Research, Massachusetts Institute of Technology, Cambridge, MA 02139, USA.

$10 \quad{ }^{4}$ Broad Institute of MIT and Harvard, Cambridge, MA 02142, USA.

$11{ }^{\dagger}$ These authors contributed equally to this work

12 *e-mail: gbchoi@mit.edu

\section{Abstract}

16 Innate social behaviors, such as mating and fighting, are fundamental to animal reproduction and

17 survival. However, social engagements can also put an individual at risk. Little is known about

18 the neural mechanisms that allow for appropriate risk assessment and the suppression of

19 hazardous social interactions. We have identified the posteromedial nucleus of the cortical

20 amygdala (COApm) as a locus required for the suppression of male mating when a female is

21 sick. Using anatomical tracing, functional imaging, and circuit-level epistatic analyses, we show

22 that suppression of mating with an unhealthy female is mediated by the COApm projections onto

23 the glutamatergic population of the medial amygdalar nucleus (MEA). We further show that the

24 role of the COApm to MEA connection in regulating male mating behavior relies on the

25 neuromodulator thyrotropin-releasing hormone (TRH). TRH is expressed in the COApm while 
26 the TRH receptor (TRHR) is found in the postsynaptic MEA glutamatergic neurons.

27 Manipulating neural activity of TRH-expressing neurons in the COApm modulated male mating

28 behavior. In the MEA, activation of the TRHR pathway, by ligand infusion, inhibited mating

29 even towards healthy females, while genetic ablation of TRHR facilitates mating with unhealthy

30 individuals. We have, therefore, uncovered a novel neural pathway that relies on the

31 neuromodulator TRH to modulate social interactions according to the health status of the

32 reciprocating individual. An individual is in need to balance the cost-benefit of social

33 interactions, with deficits in the ability to select healthy mates likely leading to the spread of

34 disease.

\section{Main}

37 Social behaviors are fundamental for survival — for the individual as well as the species. Social

38 engagements, however, also carry inherent risks, such as contracting pathogens from a sick

39 mate $^{1}$. Thus, the ability to modify innate social behaviors to account for the health status of a

40 partner is essential to minimize potential risks and to enhance survival ${ }^{2-4}$. Innate social behaviors,

41 such as mating and aggression ${ }^{5}$, are largely thought to be coordinated by developmentally

42 determined neural pathways ${ }^{6-9}$. However, the molecular and neural circuit mechanisms that

43 mediate the adaptive modulation of innate social interactions according to the health of a partner

44 are still lacking.

46 Male mice avoid mating with LPS-treated, unhealthy females

47 Mice avoid a potentially sick partner. When we presented male mice with a pair of estrus

48 females, they preferentially mounted females that were intraperitoneally injected with control 
49 phosphate-buffered saline (PBS-female) over those injected with lipopolysaccharide (LPS-

50 female), a cell wall component of gram-negative bacteria that induces an immune state that

51 mimics bacterial infection ${ }^{10}$ (Extended Data Fig. 1a-b). No such bias was observed when males

52 were given a choice between two untreated healthy females (Extended Data Fig. 1c). We

53 observed no preference for PBS- or LPS-females in a three-chamber sociability assay in which

54 females are encaged in wired cups (Extended Data Fig. 1d), suggesting that direct access to the 55 female is likely needed to establish her health status. To further examine the repertoire of male

56 mating behaviors displayed towards unhealthy females, we carried out a single-female assay, in

57 which males were presented either with a PBS- or LPS-female (Fig. 1a and Extended Data Fig.

58 1e). Males reduced the amount of anogenital investigation towards LPS-females, while

59 maintaining a comparable level of facial investigation (Extended Data Fig. 1e-f). Notably, the

60 percentage of male mice that mounted LPS-females as well as the duration and frequency of

61 mounting bouts were reduced, while the latency to mount LPS-females substantially increased

62 (Fig. 1b-e). These observations indicate that males lessen the expression of mating behaviors

63 toward LPS-treated, unhealthy females. Of note, the LPS treatment induced changes also in the

64 repertoire of female behaviors, generally reducing locomotion as quantified by the total number 65 of cage crossings (Extended Data Fig. 1g,h).

66

67 The COApm is activated in male mice in response to a LPS-treated female

68 We next carried out experiments using the single-female assay to elucidate the molecular and

69 circuit-level mechanism underlying the suppression of male mating behavior towards unhealthy,

70 LPS-females. We reasoned that brain regions that play a role in suppressing mating may be

71 specifically activated in the presence of LPS-females. Consistent with the role of the accessory 
72 olfactory system in recognizing the health of other individuals ${ }^{11-14}$, we found that surgically

73 removing the vomeronasal organ impaired active suppression of male mating behaviors towards

74 LPS-females (Extended Data Fig. 2a). Therefore, using FOS expression as a marker of neural

75 activity, we examined whether the accessory olfactory bulb (AOB) and its direct downstream

76 targets - the bed nucleus of the stria terminalis (BST), MEA, and COApm ${ }^{15,16}$ (Extended Data

77 Fig. 2b-d) - are preferentially active when a male is presented with an LPS-female compared to a

78 PBS-female. We observed the highest fold change in the number of FOS+ neurons in the

79 COApm and, to a lesser extent in the AOB and the posteroventral portion of the MEA (MEApv)

80 (Fig. 1f,g and Extended Data Fig. 2h). To directly monitor COApm neural activity in awake

81 behaving animals, we expressed the genetically encoded fluorescent calcium indicator GCaMP6s

82 and performed fiber photometry (Fig. 1h). While direct investigation of both PBS- and LPS-

83 females evoked time-locked neural activity in the COApm, the response to LPS-females was

84 more pronounced and did not readily habituate when compared to PBS-females (Fig. 1i-1 and

85 Extended Data Fig. 3a-c). Levels of neural activity in the COApm during investigation,

86 therefore, negatively correlated with the incidence of mounting (Fig. 1m). Notably, the COApm

87 remained silent during mounting (Fig. 1n,o). We did not find any observable difference in

88 COApm neural activity (Extended Data Fig. 3d,e) or males' mounting attempts (Extended Data

89 Fig. 3f-i) towards estrus versus diestrus females during a 10-min testing period. A higher

90 percentage of estrus female partners, however, were found with mating plugs $24-\mathrm{hr}$ after the

91 testing (Extended Data Fig. 3j), demonstrating their enhanced receptivity compared to diestrus

92 females. These data collectively indicated that COApm is selectively activated by the unhealthy

93 status of reciprocating females independently of their estrus phase. 
94 COApm is a direct target of the AOB (Extended Data Fig. 2b-g) suggesting that the suppression

95 of mating behaviors induced by LPS-females might be dependent on non-volatile odorants. We

96 thus used urine and feces of LPS-females as odor sources to test if odor alone can substitute for

97 an LPS-female. Indeed, LPS-odor induced a greater number of FOS+ neurons in the COApm

98 than PBS-odor (Extended Data Fig. 4a-c). A similar observation was made using fiber

99 photometry (Extended Data Fig. 4d,e). LPS-odor painted on healthy females resulted in a

100 decrease in both mounting and anogenital investigation of otherwise healthy females (Extended

101 Data Fig. 4f-k). Importantly, LPS-odor suppressed male mating behaviors but did not alter the

102 behaviors of the odor-presenting females (Extended Data Fig. 41,m), demonstrating that

103 suppression of male sexual behaviors depends largely on the olfactory bouquet of the female

104 rather than the behavioral changes that follow LPS treatment.

105

\section{COApm suppresses male mating}

107 The observation that COApm preferentially responds to LPS-females while remaining silent

108 during mounting suggested that it might be directly involved in suppressing male mating

109 behaviors in the presence of unhealthy partners. Consistent with this notion, optogenetic

110 activation of COApm neurons robustly inhibited mounting even towards healthy females (Fig.

111 2a-e, Extended Data Fig.5a). COApm photoactivation, however, did not affect the total

112 investigation time directed towards females (Extended Data Fig. 5b) or the expression of other

113 motivational behaviors, such as feeding and sociability (Fig. 2f,g). Mice also showed neither

114 aversion nor attraction for the side of the chamber where COApm was activated during a real-

115 time place preference test (RTPP) (Fig. 2h). We noted that COApm photoactivation increased

116 self-grooming. However, this increase could at best account for a small fraction of the total 
117 reduction in mounting, and the fidelity with which the COApm stimulation induced grooming

118 was low (Extended Data Fig. 5c,d). In accord with the results from the photoactivation

119 experiments, inhibition of the COApm via bilateral expression of the inhibitory DREADD

120 hM4Di (Fig. 2i and Extended Data Fig. 5e) significantly increased male mounting behavior

121 towards LPS-females, with male mice exhibiting both heightened duration and frequency of

122 mounting (Fig. 2j-1 and Extended Data Fig. 5g-j). The latency to the first mounting attempt

123 showed a trend towards a decrease, although the duration of direct investigation remained

124 unchanged (Fig. 2m and Extended Data Fig. 5f,k). Inhibition of COApm did not affect mounting

125 towards untreated, healthy females regardless of the phase of the estrus cycle (Extended Data

126 Fig. 51-u). Taken together, these results demonstrate that the COApm has a critical role in the

127 suppression of mating behaviors when male mice are faced with unhealthy females.

\section{COApm-mediated suppression of mating requires the MEA glutamatergic neurons}

130 To gain further insights into the circuit-level mechanisms that underlie COApm suppression of

131 social behaviors, we performed anterograde tracing experiments to identify downstream targets

132 of the COApm. Among the labeled regions, the MEA, known to be involved in regulating innate

133 social behaviors ${ }^{17-20}$, receives the largest COApm axonal projection (Extended Data Fig. 6a,b).

134 The majority of MEA neurons postsynaptic to the COApm are located in the MEApv, which is

135 enriched for Vglut2(+) glutamatergic neurons over Vgat(+) GABAergic neurons (Extended Data

136 Fig. 6c-g). We confirmed the functional relevance of the COApm-to-MEA ${ }^{\text {Vglut2+ }}$ projection by

137 monitoring the activity of the Vglut2(+) or Vgat(+) populations in response to optogenetic

138 activation of the COApm. Stimulating the COApm evoked responses preferentially in MEA-

139 Vglut2(+) neurons as measured using both GCaMP fluorescence (Fig. 3a-e). Moreover, MEA- 
Vglut2(+) neurons, like the COApm, were directly activated by LPS-females (Extended Data

141 Fig. 6h,i). These data suggest that the COApm suppresses mating behavior towards LPS-females

142 by engaging the MEA-Vglut2(+) population. Indeed, optogenetic stimulation of the COApm

143 axonal terminals in the MEA inhibited mating (Fig. 3f) similarly to the stimulation of COApm

144 neuronal cell bodies (Fig. 2a-e). Activation of the MEA-Vglut2(+) population also suppressed

145 mating behavior (Fig. 3g). Conversely, inhibiting the MEA-Vglut2(+) population impaired the

146 natural ability of the males to suppress mating towards an unhealthy LPS-female (Fig. 3h).

147 Furthermore, using circuit-level functional epistasis experiments, we demonstrated that

148 activation of the COApm axonal terminals, while concurrently inhibiting the MEA-Vglut2(+)

149 population, fails to suppress mating behavior (Fig. 3i, also see Extended Data Fig. 7), suggesting

150 that the COApm-to-MEA ${ }^{\text {Vglut2+ }}$ projection is required to modulate male mouse mating behaviors

151 on the basis of the perceived health status of the female.

\section{Suppression of mating towards unhealthy females engages COApm-TRH(+) neurons}

154 Determining the distinctive transcriptional profile of COApm neurons may provide insight on the

155 genetic basis that allow the COApm to regulate social interactions. To this end, we used viral

156 strategies ${ }^{21}$ to label the subset of COApm neurons that specifically project to the MEA-Vglut2(+)

157 neurons, with a GFP-tagged version of the ribosomal protein L10a (Extended Data Fig. 8a).

158 Following Translating Ribosome Affinity Purification (TRAP) ${ }^{22}$, we performed RNA sequencing

159 and differential gene expression analyses, identifying 1242 differentially expressed genes that are

160 highly enriched in the COApm population that projects to MEA-Vglut2(+) neurons (Extended

161 Data Fig. 8b). Of those, we selected the tripeptide molecule thyrotropin-releasing hormone

162 (TRH) - one of the most highly enriched candidates belonging to the neuroactive ligand-receptor 
163 interaction pathway - for further analyses (Extended Data Fig. 8c). In situ hybridization

164 confirmed that $\operatorname{Trh}$ is specifically and selectively expressed in the COApm (Fig. 4a,b). We next

165 functionally tested the role of $\mathrm{COApm}-\mathrm{TRH}(+)$ neurons in suppressing male mating behaviors.

166 Selective activation of TRH(+) neurons robustly suppressed mounting towards healthy females

167 (Fig. 4c-h). On the other hand, photostimulation of TRH(-) cells did not suppress mounting

168 behavior, indicating that the ability to modulate mating is restricted to $\mathrm{TRH}(+)$ neurons in the

169 COApm (Extended Data Fig. 9a-g). While Trh is expressed by COApm neurons, the TRH

170 receptor (TRHR) is selectively enriched in Vglut2+ MEA neurons (Fig. 4i,j), suggesting that

171 TRH may modulate expression of innate social behaviors by acting on the COApm-MEA ${ }^{\text {vglut2+ }}$

172 circuit. Consistent with this hypothesis, direct delivery of the TRHR agonist taltirelin ${ }^{23}$ into the

173 MEA evoked robust responses in Vglut2+ neurons in vitro (Extended Data Fig. 9h-l) and

174 suppressed mating towards healthy females (Fig. 4k-o and Extended Data Fig. 9m). To

175 determine whether TRHR signaling in the MEA is involved in modulating mating behaviors

176 according to the health status of females, we generated a TRHR conditional knock-out mouse

177 line (Extended Data Fig. 10a). Genetic removal of the TRHR, by delivering Cre-expressing virus

178 into the MEA, reduced neural activity evoked by photoactivation of COApm-MEA projections

179 (Extended Data Fig. 10b-g) and impaired a male's ability to suppress mating towards LPS-

180 females (Fig. 4p-t). These data collectively indicate that suppression of mating behavior towards

181 an unhealthy mate depends on the activity of the COApm-MEA ${ }^{\mathrm{Vglut} 2+}$ projection expressing

182 TRH-TRHR.

183

184 Discussion 
185 While an increasing number of studies suggests that sick individuals engage in a repertoire of

186 sickness behaviors to limit their social interactions ${ }^{24-27}$, little is known about how healthy

187 individuals respond to an infected conspecific. Here, we have identified an amygdalar circuit

188 responsible for discouraging innate reproductive social behaviors if a male encounters a sick

189 partner. We show that the odor bouquet of a sick female is sufficient to activate the COApm and

190 inhibit male mating behaviors, suggesting odorants associate with the "sickness state" of the

191 females can be smelled by males, using odorant receptors like the family of the formyl peptide

192 receptors ${ }^{12,13}$. Posteroventral MEA-Vglut2(+) neurons, postsynaptic to the COApm, are essential

193 for the modulation of mating behaviors to unhealthy females. The posterodorsal MEA-Vglut2(+)

194 neurons have been previously shown to have a similar role in inhibiting innate social

195 behaviors ${ }^{17,20}$, suggesting that Vglut2(+) neurons in the dorsal and ventral parts of the posterior

196 MEA could comprise a functional unit partaking in the regulation of social behaviors. Our data

197 also suggest that the COApm-MEA ${ }^{\text {Vglut2+ }}$ projection relies on TRH to modulate reproductive

198 social behaviors. Of note, thyroid dysfunction and dysregulated levels of thyroid hormones have

199 been associated with depression and social anxiety in humans ${ }^{28,29}$. Whether TRH has a role in the

200 expression of these symptoms remains to be explored. Overall, our data characterized a novel

201 neural node that takes into account the health status of the interacting partner to modulate innate

202 mating behavior. As limiting the spread of a pathogen likely requires the concerted efforts of

203 both infected and uninfected individuals, the COApm-MEA ${ }^{\mathrm{Vglut} 2+}$ circuit we have identified is

204 likely to contribute to the wellness of an individual as well as its community. It is of paramount

205 importance for an individual to maintain a balance between the drive to socially interact while

206 minimizing risks through social avoidance. 


\section{References}

2091 Altizer, S. et al. Social Organization and Parasite Risk in Mammals: Integrating Theory and Empirical Studies. Annual Review of Ecology, Evolution, and Systematics 34, 517547 (2003).

2 Hart, B. L. Behavioral adaptations to pathogens and parasites: five strategies. Neuroscience and biobehavioral reviews 14, 273-294, doi:10.1016/s01497634(05)80038-7 (1990).

3 Ehman, K. D. \& Scott, M. E. Female mice mate preferentially with non-parasitized males. Parasitology 125, 461-466, doi:10.1017/s003118200200224x (2002).

4 Chen, P. \& Hong, W. Neural Circuit Mechanisms of Social Behavior. Neuron 98, 16-30, doi:10.1016/j.neuron.2018.02.026 (2018).

5 Tinbergen, N. The study of instinct. New York, NY: Claredon Press/Oxford University Press (1951).

6 Chamero, P. et al. Identification of protein pheromones that promote aggressive behaviour. Nature 450, 899-902, doi:10.1038/nature05997 (2007).

7 Hashikawa, K., Hashikawa, Y., Falkner, A. \& Lin, D. The neural circuits of mating and fighting in male mice. Current opinion in neurobiology 38, 27-37, doi:10.1016/j.conb.2016.01.006 (2016).

8 Stowers, L., Holy, T. E., Meister, M., Dulac, C. \& Koentges, G. Loss of sex discrimination and male-male aggression in mice deficient for TRP2. Science 295, 1493-1500, doi:10.1126/science.1069259 (2002).

9 Leypold, B. G. et al. Altered sexual and social behaviors in trp2 mutant mice. Proceedings of the National Academy of Sciences of the United States of America 99, 6376-6381, doi:10.1073/pnas.082127599 (2002).

10 Medzhitov, R. Toll-like receptors and innate immunity. Nature reviews. Immunology $\mathbf{1}$, 135-145, doi:10.1038/35100529 (2001).

11 Boillat, M. et al. The vomeronasal system mediates sick conspecific avoidance. Current biology : $C B$ 25, 251-255, doi:10.1016/j.cub.2014.11.061 (2015).

12 Riviere, S., Challet, L., Fluegge, D., Spehr, M. \& Rodriguez, I. Formyl peptide receptorlike proteins are a novel family of vomeronasal chemosensors. Nature 459, 574-577, doi:10.1038/nature08029 (2009).

13 Liberles, S. D. et al. Formyl peptide receptors are candidate chemosensory receptors in the vomeronasal organ. Proceedings of the National Academy of Sciences of the United States of America 106, 9842-9847, doi:10.1073/pnas.0904464106 (2009).

14 Kavaliers, M., Choleris, E., Agmo, A. \& Pfaff, D. W. Olfactory-mediated parasite recognition and avoidance: linking genes to behavior. Hormones and behavior 46, 272283, doi:10.1016/j.yhbeh.2004.03.005 (2004).

15 Scalia, F. \& Winans, S. S. The differential projections of the olfactory bulb and accessory olfactory bulb in mammals. The Journal of comparative neurology 161, 31-55, doi:10.1002/cne.901610105 (1975).

16 Boehm, U. The vomeronasal system in mice: from the nose to the hypothalamus- and back! Seminars in cell \& developmental biology 17, 471-479, doi:10.1016/j.semcdb.2006.04.013 (2006).

17 Choi, G. B. et al. Lhx6 delineates a pathway mediating innate reproductive behaviors from the amygdala to the hypothalamus. Neuron 46, 647-660, doi:10.1016/j.neuron.2005.04.011 (2005).

$18 \mathrm{Li}$, Y. et al. Neuronal Representation of Social Information in the Medial Amygdala of Awake Behaving Mice. Cell 171, 1176-1190 e1117, doi:10.1016/j.cell.2017.10.015 (2017). 
25719 Ishii, K. K. et al. A Labeled-Line Neural Circuit for Pheromone-Mediated Sexual

258 Behaviors in Mice. Neuron 95, 123-137 e128, doi:10.1016/j.neuron.2017.05.038 (2017). Hong, W., Kim, D. W. \& Anderson, D. J. Antagonistic control of social versus repetitive self-grooming behaviors by separable amygdala neuronal subsets. Cell 158, 1348-1361, doi:10.1016/j.cell.2014.07.049 (2014).

21 Wickersham, I. R., Finke, S., Conzelmann, K. K. \& Callaway, E. M. Retrograde neuronal tracing with a deletion-mutant rabies virus. Nature methods 4, 47-49, doi:10.1038/nmeth999 (2007).

22 Heiman, M. et al. A translational profiling approach for the molecular characterization of CNS cell types. Cell 135, 738-748, doi:10.1016/j.cell.2008.10.028 (2008).

23 Suzuki, M., Sugano, H., Matsumoto, K., Yamamura, M. \& Ishida, R. Synthesis and central nervous system actions of thyrotropin-releasing hormone analogues containing a dihydroorotic acid moiety. Journal of medicinal chemistry 33, 2130-2137, doi:10.1021/jm00170a014 (1990).

24 Hart, B. L. Biological basis of the behavior of sick animals. Neuroscience and biobehavioral reviews 12, 123-137, doi:10.1016/s0149-7634(88)80004-6 (1988).

25 Lopes, P. C., Block, P. \& Konig, B. Infection-induced behavioural changes reduce connectivity and the potential for disease spread in wild mice contact networks. Scientific reports 6, 31790, doi:10.1038/srep31790 (2016).

26 Van Kerckhove, K., Hens, N., Edmunds, W. J. \& Eames, K. T. The impact of illness on social networks: implications for transmission and control of influenza. American journal of epidemiology 178, 1655-1662, doi:10.1093/aje/kwt196 (2013).

27 Stockmaier, S., Bolnick, D. I., Page, R. A. \& Carter, G. G. Sickness effects on social interactions depend on the type of behaviour and relationship. The Journal of animal ecology, doi:10.1111/1365-2656.13193 (2020).

28 Fischer, S. \& Ehlert, U. Hypothalamic-pituitary-thyroid (HPT) axis functioning in anxiety disorders. A systematic review. Depression and anxiety 35, 98-110, doi:10.1002/da.22692 (2018).

29 Baumgartner, A. Thyroxine and the treatment of affective disorders: an overview of the results of basic and clinical research. The international journal of neuropsychopharmacology 3, 149-165, doi:10.1017/S1461145700001887 (2000).

30 Krashes, M. J. et al. An excitatory paraventricular nucleus to AgRP neuron circuit that drives hunger. Nature 507, 238-242, doi:10.1038/nature12956 (2014).

31 Chen, T. W. et al. Ultrasensitive fluorescent proteins for imaging neuronal activity. Nature 499, 295-300, doi:10.1038/nature12354 (2013).

32 Zhang, F. et al. Optogenetic interrogation of neural circuits: technology for probing mammalian brain structures. Nature protocols 5, 439-456, doi:10.1038/nprot.2009.226 (2010).

33 Ung, K. \& Arenkiel, B. R. Fiber-optic implantation for chronic optogenetic stimulation of brain tissue. Journal of visualized experiments : JoVE, e50004, doi:10.3791/50004 (2012).

34 Armbruster, B. N., Li, X., Pausch, M. H., Herlitze, S. \& Roth, B. L. Evolving the lock to fit the key to create a family of $G$ protein-coupled receptors potently activated by an inert ligand. Proceedings of the National Academy of Sciences of the United States of America 104, 5163-5168, doi:10.1073/pnas.0700293104 (2007).

35 Pankevich, D. E., Baum, M. J. \& Cherry, J. A. Olfactory sex discrimination persists, whereas the preference for urinary odorants from estrous females disappears in male mice after vomeronasal organ removal. The Journal of neuroscience : the official journal of the Society for Neuroscience 24, 9451-9457, doi:10.1523/JNEUROSCI.2376-04.2004 (2004). 
36 McClure, C., Cole, K. L., Wulff, P., Klugmann, M. \& Murray, A. J. Production and titering

\section{Supplementary Information}

344 Supplementary statistical information is attached.

\section{Acknowledgements}

347 We thank N. Soares, M. Andina, K. Ronayne and I.D. Mejia for assistance with experiments and 
349 generously sharing the $\operatorname{Trh}$-Cre mouse line. We thank H. Choi for the fiber photometry set-up.

350 This work was supported by the National Institute of Mental Health grants R01 MH122270 and 351 R01 MH106497 (G.B.C.), the JPB Foundation (M.H. and G.B.C), Simons Center for the Social 352 Brain Postdoctoral Fellowship (J.K. and H.K.C) and the Picower Fellows (J.K. and H.L.).

\section{Author Contributions}

355 J.K. and G.B.C. conceptualized the study. J.K., H.L., C.R., M.H. and G.B.C. designed the 356 experiments and/or provided advice and technical expertise. J.K., H.L., C.R., A.S., J.F., D.H.C., 357 S.B., H.S., H.K.C. performed the experiments. I.R.W. provided reagents. J.K. and G.B.C. wrote 358 the manuscript with inputs from the co-authors.

360 Author Information The authors declare no competing financial interests. Correspondence and 361 requests for materials should be addressed to G.B.C. (gbchoi@ mit.edu). 
a
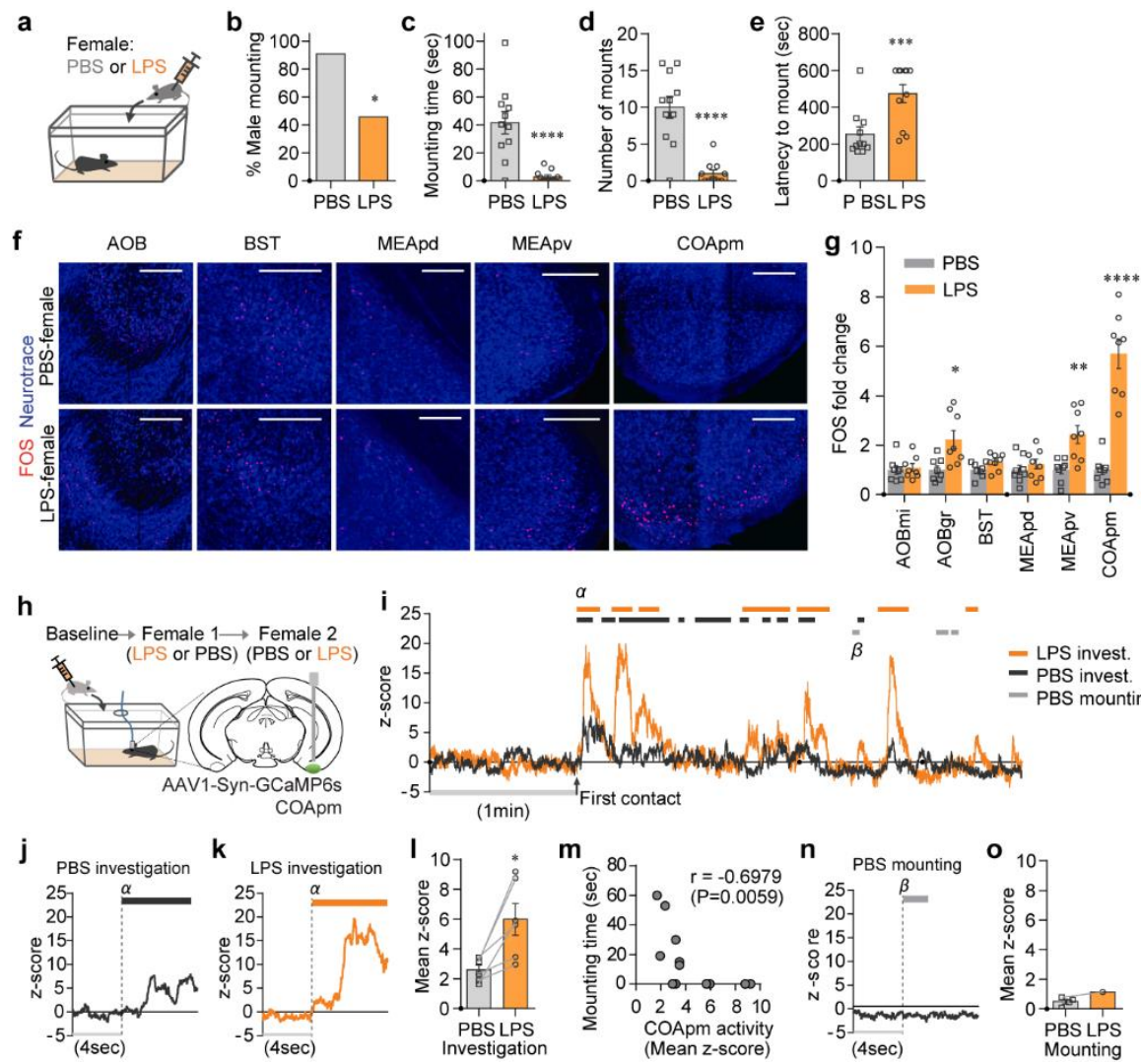

Figure 1. COApm is activated by LPS-treated females. a-e, Male mounting behaviors towards

a PBS- or LPS-treated female. Percentage of males engaged in mounting behavior (percent male mounting) (b), total duration of mounting bouts (mounting time) (c), number of mounting

367 attempts (number of mounts) (d) and latency to the first mounting attempt (latency to mount) (e) during a 10-min test (PBS, $n=11$ and LPS, $n=11$; from 2 independent experiments). $\mathbf{f , g}$

369 Representative images of FOS expression in the vomeronasal pathway after interaction with

370 PBS- or LPS-females (f) and fold change in the number of FOS-expressing neurons normalized

371 to the mean of the PBS-female group for each region (g) (PBS, $n=8$ and LPS, $n=8$; from 2

372 independent experiments). Scale bar $=200 \mu \mathrm{m}$. AOBmi: mitral layer of the accessory olfactory

373 bulb, AOBgr: granular layer of the accessory olfactory bulb, BST: bed nucleus of the stria

374 terminalis, MEApd: posterodorsal part of the medial amygdalar nucleus, MEApv: posteroventral 
375 part of the medial amygdalar nucleus. h-o, Virus encoding GCaMP6s (AAV ${ }_{1}$-Syn-GCaMP6s)

376 was targeted to the COApm for fiber photometry recordings. Male mice were sequentially

377 presented with a PBS- or LPS-female in counterbalanced sessions (h). Representative trace of

378 COApm bulk fluorescence signal during interactions with the PBS- (black) or LPS-female

379 (orange) (i). Representative traces (marked as $\alpha$ in (i)) (j,, $\mathbf{k})$ and the average mean z-score of the

380 fluorescence during direct investigation of the PBS- or LPS-female (I). Spearman's correlation

381 between mean z-score during investigation and mounting time. Data are pulled from the sessions

382 with PBS- and LPS-females (m). Representative trace (marked as $\beta$ in (i)) (n) and the average

383 mean z-score of the fluorescence during mounting of the PBS- or LPS-female (o) ( $n=6$ from 3

384 independent experiments). $* P<0.05, * * P<0.01, * * * P<0.001$ and $* * * * P<0.0001$ calculated by

385 Chi-Square Test of Independence (b), unpaired two-tailed $t$-test (c-e), two-way ANOVA with

386 Sidak's post-hoc test (g) and paired two-tailed $t$-test (l). Graphs indicate mean \pm s.e.m. p-values

387 are described in the supplementary statistical information file. 

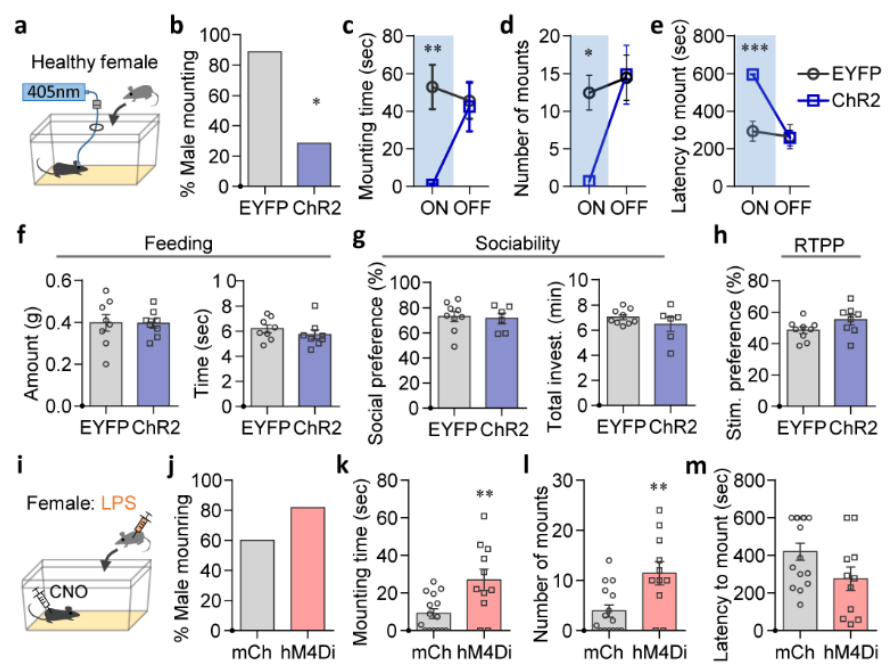

Figure 2. COApm mediates suppression of mating behaviors towards unhealthy females. a-

e, Male mice expressing EYFP ( $\mathrm{AAV}_{2}$-hSyn-EYFP) or channelrhodopsin (ChR2, $\mathrm{AAV}_{2}$-hSyn-

hChR2-EYFP) in COApm were tested for mating behaviors towards healthy females in the presence $(\mathrm{ON})$ or absence $(\mathrm{OFF})$ of COApm photoactivation. Percent male mounting $(\mathbf{b})$, mounting time (c), number of mounts (d), and latency to mount (e) (EYFP, $n=9$ and ChR2, $n=7$; from 2 independent experiments). f, Total amount of food eaten and time spent eating used to assess feeding behavior during COApm photoactivation (EYFP, $n=8$ and ChR2, $n=8$; from 2 independent experiments). g, Social preference (time spent investigating a social object/total

398 time spent investigating social and inanimate objects) and total investigation time measured 399 during a sociability test with COApm photoactivation (EYFP, $n=9$ and ChR2, $n=6$; from 2

400 independent experiments). $\mathbf{h}$, Stimulation preference (time spent in the compartment where 401 photoactivation occurs/total testing time) during a real-time place preference (RTPP) test (EYFP, $402 n=9$ and $\mathrm{ChR} 2, n=8$; from 2 independent experiments). i-m, Male mice expressing mCherry

403 (AAV 2 -hSyn-mCherry) or inhibitory DREADD (hM4Di, AAV 2 -hSyn-hM4D(Gi)-mCherry) in 404 COApm were injected with Clozapine-N-Oxide (CNO) and tested for mating behaviors towards 
405 LPS-females (i). Percent male mounting (j), mounting time (k), number of mounts (l), and 406 latency to mount (m) (mCherry, $n=15$ and hM4Di, $n=11$; from 3 independent experiments). $407 * P<0.05, * * P<0.01$ and $* * * P<0.001$ calculated by Chi-Square Test of Independence (b), two408 way repeated measures ANOVA with Bonferroni's post-hoc test (c-e) and unpaired two-tailed $t$ 409 test $(\mathbf{k}, \mathbf{l})$. Graphs indicate mean \pm s.e.m. p-values are described in the supplementary statistical 410 information file. 
a
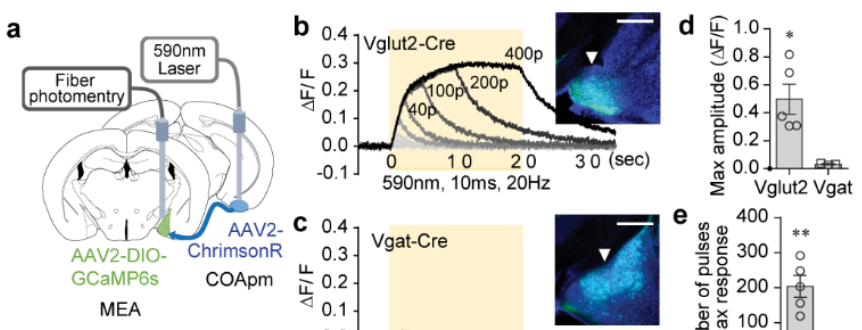

Vglut2- or Vgat-Cre mice
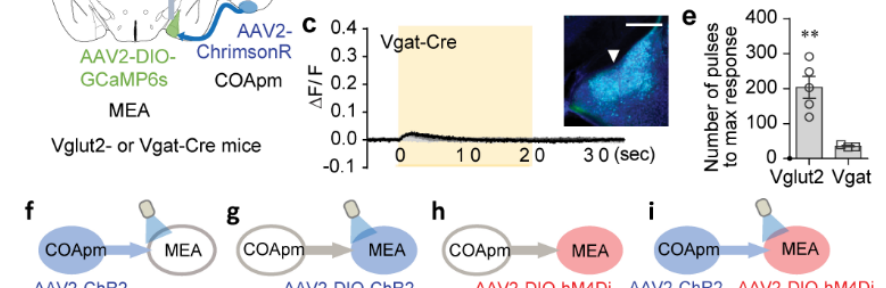

h $\quad$ i
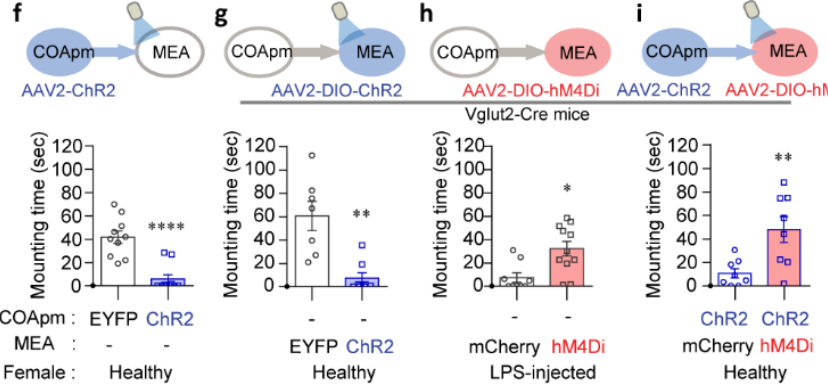

Figure 3. COApm projections to MEA-Vglut2(+) neurons mediate suppression of mating

towards LPS-females. a-e, Red-shifted opsin ChrimsonR ( $\mathrm{AAV}_{2}$-hSyn-ChrimsonR-tdTomato)

was expressed in COApm, while GCaMP6s was expressed in MEA for fiber photometry

analyses. Bulk fluorescence signal upon COApm photoactivation was measured in either MEA-

417 Vglut2(+) or MEA-Vgat(+) neurons. Representative traces of changes in fluorescence signal in

418 MEA upon photoactivation of COApm using light trains with 1, 5, 10, 20, 40, 100, 200 and 400

419 pulses, respectively. Inset images show GCaMP6s expression in MEA-Vglut2(+) or MEA-

420 Vgat(+) neurons. Arrowheads indicate the placement of the optic fiber for fiber photometry (b,c).

421 Maximum amplitude evoked by photoactivation of COApm with 400 pulses of light (d) and

422 number of light pulses to reach max amplitude (e) (Vglut2-Cre, $n=5$ and Vgat-Cre, $n=3$; from 3

423 independent experiments). Scale bar $=500 \mu \mathrm{m}$. f-i, Quantification of male mounting behaviors

424 during concurrent manipulation of COApm-MEA axonal projections and MEA-Vglut2(+)

425 neurons. Male subjects were wild type for (f) or Vglut2-Cre mice for ( $\mathrm{g}$, h and i). Female

426 subjects were healthy for (f, g and i) or LPS-injected for (h). f, Mounting time with 
427 photoactivation of COApm-MEA projections (EYFP, $n=10$ and ChR2, $n=10$; from 2 independent 428 experiments). g, Mounting time with photoactivation of MEA-Vglut2(+) neurons (EYFP, $n=7$ 429 and ChR2, $n=8$; from 2 independent experiments). $\mathbf{h}$, Mounting time towards LPS-females with 430 hM4Di-inhibition of MEA-Vglut2(+) neurons (mCherry, $n=9$ and hM4Di, $n=11$; from 2

431 independent experiments). i, Mounting time with concurrent photoactivation of COApm-MEA 432 projections and hM4Di-inhibition of MEA-Vglut2(+) neurons (mCherry, $n=8$ and hM4Di, $n=8$;

433 from 2 independent experiments). $* P<0.05, * * P<0.01$ and $* * * * P<0.0001$ calculated by unpaired 434 two-tailed $t$-test. Graphs indicate mean \pm s.e.m. p-values are described in the supplementary 435 statistical information file. 

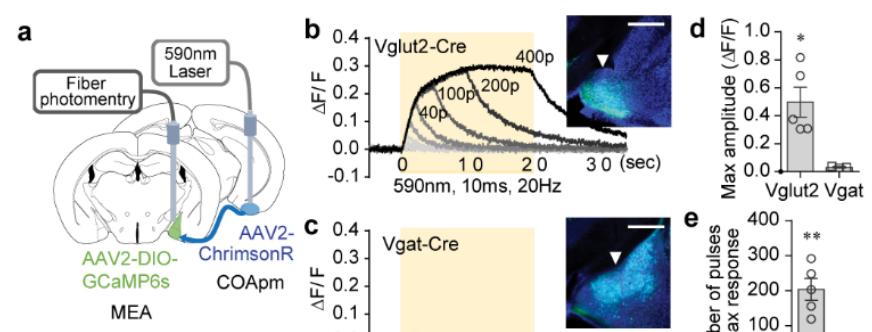

Vglut2- or Vgat-Cre mice
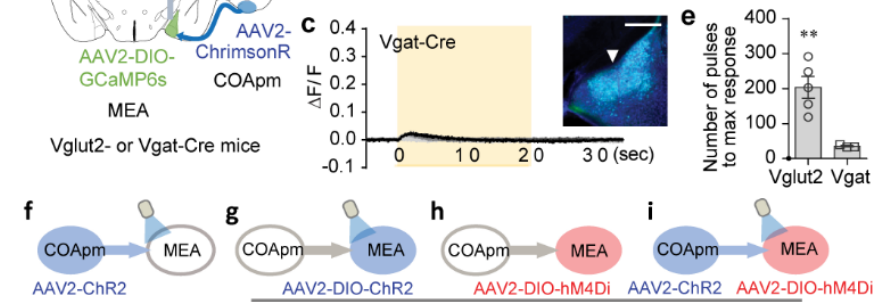

AAV2-ChR2
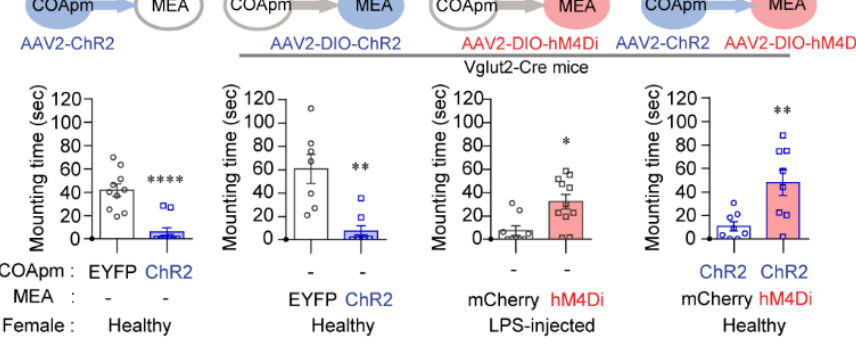

Figure 4. Suppression of social behaviors engages COApm-TRH(+) neurons. a,b,

440 Representative image (a) and quantification (b) of Trh mRNA expression in wild-type mice at

441 anterior-posterior (AP) $-2.9 \mathrm{~mm}$ according to cortical layers ( $\mathrm{n}=13$ sections from 4 mice; from 2

442 independent experiments). Dotted line defines cortical layers I, II, and III. Scale bar=1mm (left)

443 and $300 \mu \mathrm{m}$ (right). c-h, Trh-Cre male mice expressing EYFP (AAV 2 -hSyn-DIO-EYFP) or ChR2

444 (AAV2-hSyn-DIO-hChR2-EYFP) in COApm-TRH(+) neurons were tested for mating behaviors

445 towards healthy females in the presence $(\mathrm{ON})$ or absence $(\mathrm{OFF})$ of photoactivation (c).

446 Representative image of ChR2 expression in COApm (d). Percent male mounting (e), mounting 447 time (f), number of mounts (g), and latency to mount (h) (EYFP, n=13 and ChR2, n=10; from 2

448 independent experiments). Scale bar $=500 \mu \mathrm{m} . \mathbf{i}, \mathbf{j}$, Representative images (i) and quantification (j)

449 of the overlap between cells expressing Trhr (red), Vglut2 (green) and Vgat (blue) mRNA in

450 MEA of wild-type mice at AP $-1.8 \mathrm{~mm}(n=10$ MEA sections from total 5 mice; from 4

451 independent experiments). Dotted line indicates MEA. Scale bar=500 $\mu \mathrm{m}$. k-o, TRH analog

452 taltirelin was bilaterally injected into MEA (k). Percent male mounting (l), mounting time $(\mathbf{m})$, 
453 number of mounts (n), and latency to mount (o) (PBS, n=14 and Taltirelin, n=15; from 2

454 independent experiments). p-t, Wild-type (WT) or TRHR ${ }^{\text {fl/fl }}$ male mice expressing GFP (AAV $1^{-}$

455 hSyn-GFP) or Cre (AAV $1-S y n-C r e)$ in the MEA were tested for mating behaviors towards LPS-

456 females. Representative image of Cre expression in MEA (p). Percent male mounting (q),

457 mounting time (r), number of mounts (s), and latency to mount (t) (WT:Cre, $\mathrm{n}=11$;

$458 \mathrm{TRHR}^{\mathrm{fl} / \mathrm{fl}}: \mathrm{EYFP}, \mathrm{n}=8$ and $\mathrm{TRHR}^{\mathrm{fl} / \mathrm{fl}}: \mathrm{Cre}, \mathrm{n}=12$; from 2 independent experiments). Scale bar=

$459500 \mu \mathrm{m} . * P<0.05, * * P<0.01, * * * P<0.001$ and $* * * * P<0.0001$ calculated by Chi-Square Test of

460 Independence (e,l,q), two-way repeated measures ANOVA with Bonferroni's post-hoc test (f-h)

461 and paired two-tailed $t$-test (j), unpaired two-tailed $t$-test (m-o) and one-way ANOVA with

462 Bonferroni's post-hoc test (r-t). Graphs indicate mean \pm s.e.m. p-values are described in the

463 supplementary statistical information file. 

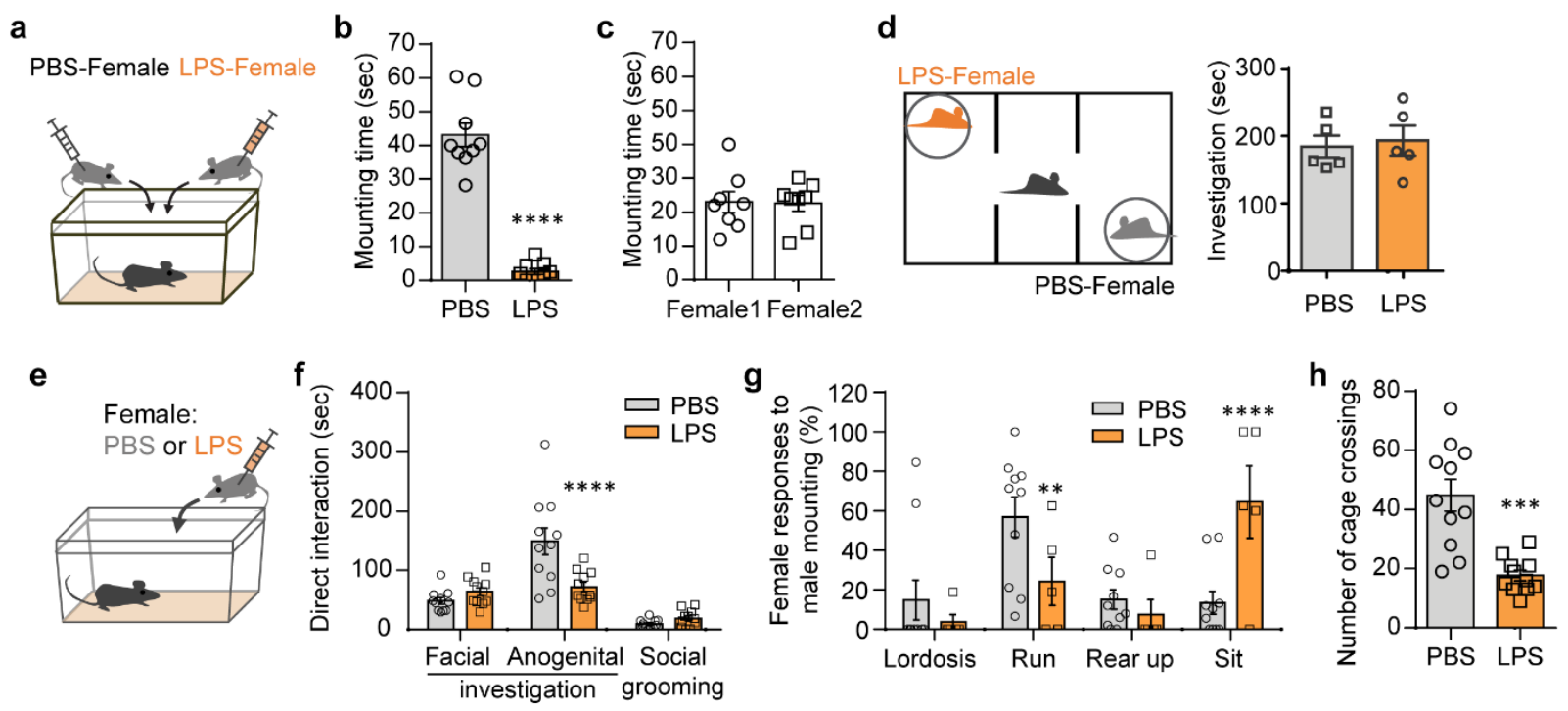

i

PBS
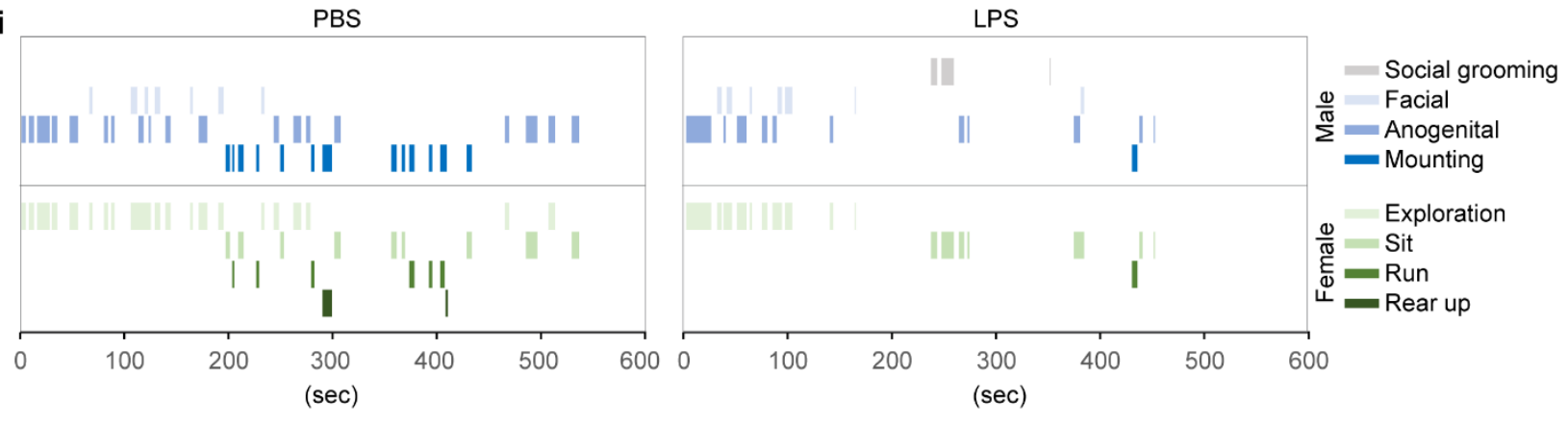

Extended Data Figure 1. Male mice avoid mounting sick females. a,b, Male mice were

467 presented with a pair of estrus females each injected intraperitoneally with either PBS (PBS-

468 female) or LPS (LPS-female) (a). Mounting time during a 10-min test (b) ( $n=9$; from 2

469 independent experiments). c, Mounting time for male mice presented with two untreated, healthy

470 females ( $n=8$; from 2 independent experiments). d, Investigation time of PBS- and LPS-females

471 during a 10-min three-chamber assay ( $n=5$; from 2 independent experiments). e-i, These data are

472 associated with Fig.1a-e. Duration of other typical male behaviors while engaged in direct

473 interactions with a PBS- or LPS- female (e,f). Percentage of individual female behaviors during

474 males' mounting attempts (g) and the number of cage crossings (h). Representative traces of

475 male and female behaviors during direct interactions (i). $* * P<0.01$, $* * * P<0.001$ and 
$476 * * * * P<0.0001$ calculated by paired two-tailed $t$-test (b), two-way ANOVA with Sidak's post-

477 hoc test (f,g) and unpaired two-tailed $t$-test (h). Graphs indicate mean \pm s.e.m. p-values are 478 described in the supplementary statistical information file. 


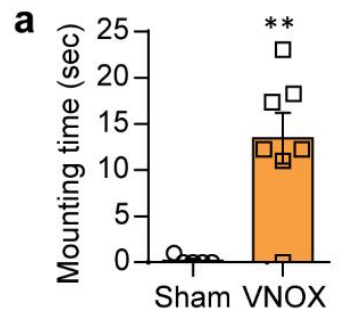

b AAV1-hSyn-Cre
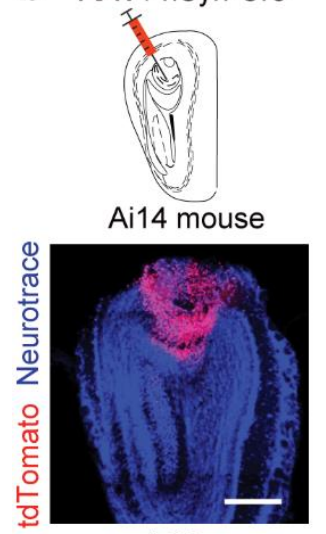

$\mathrm{AOB}$

C

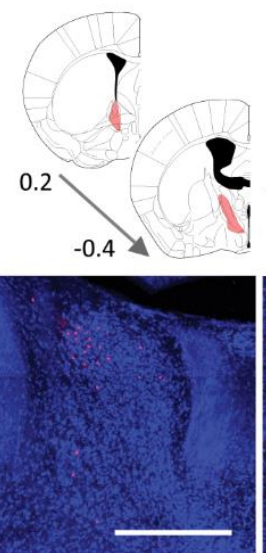

BST

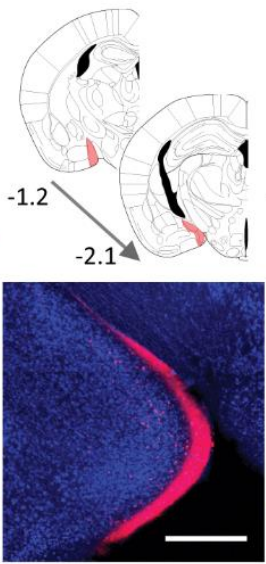

MEA

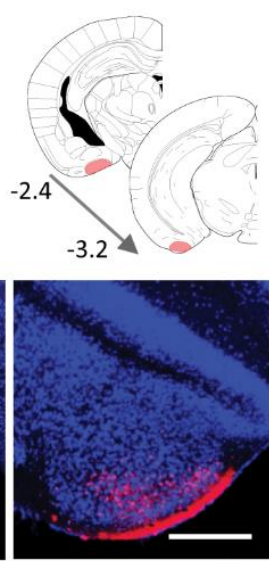

COApm
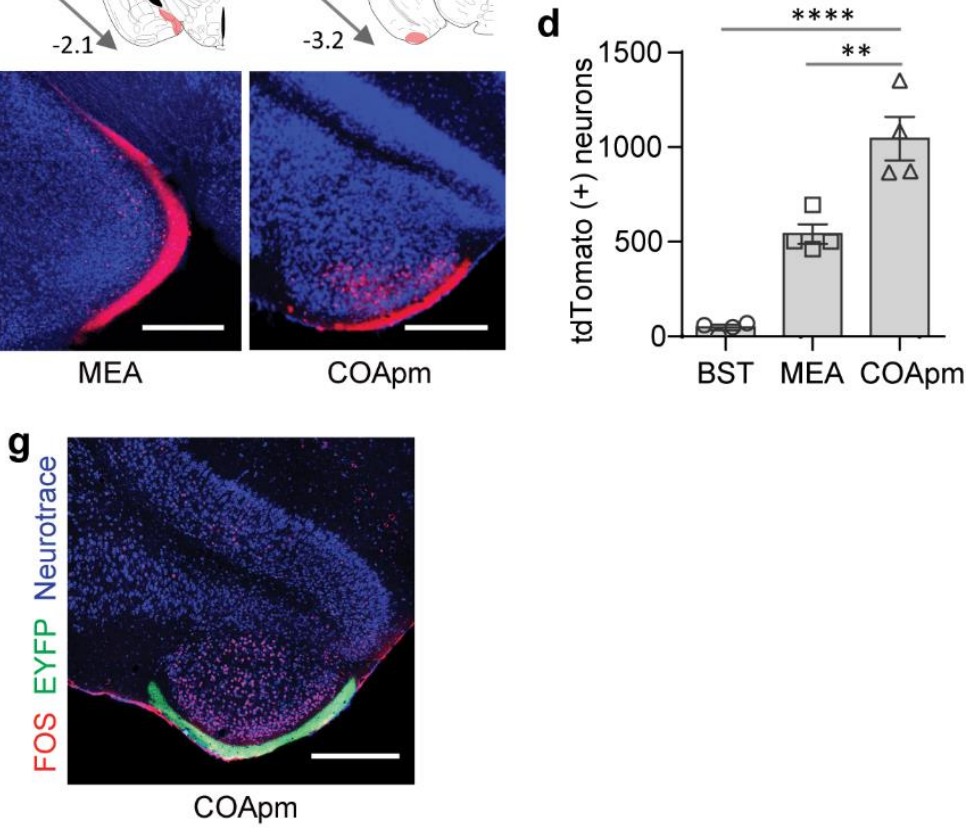

COApm

e $405 \mathrm{~nm}$ laser AAV2-hSyn-
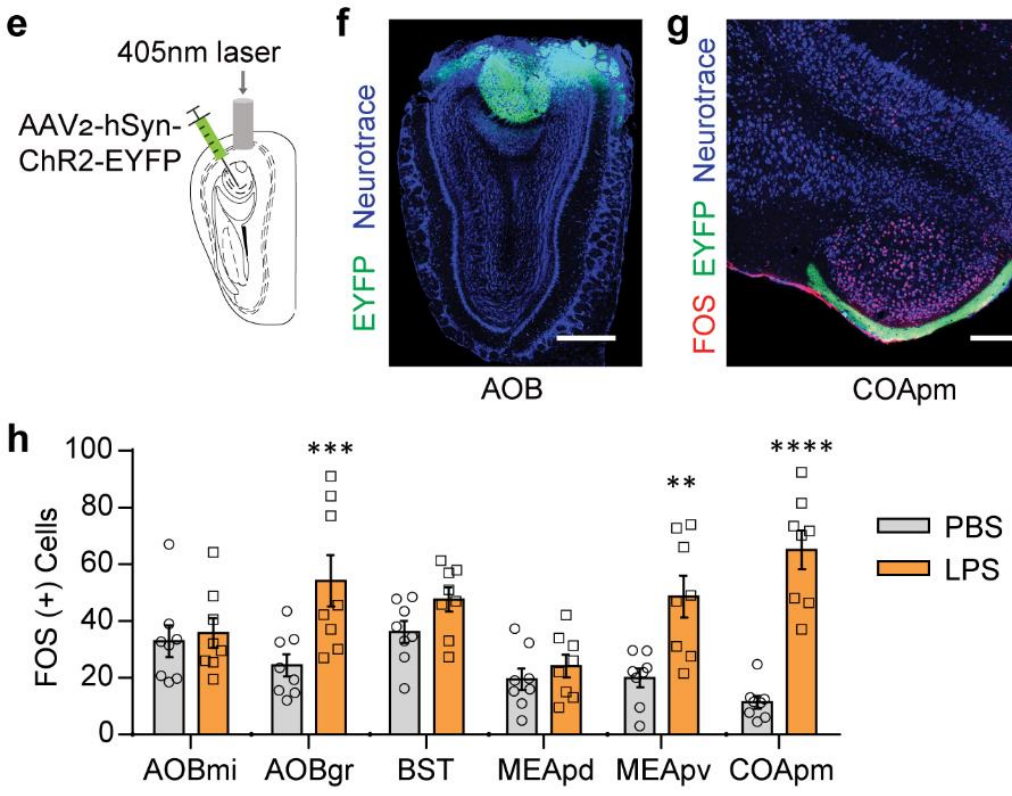

481 Extended Data Figure 2. Role of the vomeronasal pathway in mounting behavior. a,

482 Mounting time for males with a sham surgery (Sham) or the VNO removed (VNOX) towards

483 LPS-females (Sham, $n=5$ and VNOX, $n=7$; from 2 independent experiments). b-d, Virus

484 encoding the anterograde trans-synaptic tracer $\left(\mathrm{AAV}_{1}-\mathrm{hSyn}-\mathrm{Cre}\right)$ was targeted to the accessory 
485 olfactory bulb (AOB) in Ai14 reporter mice that express tdTomato in a Cre-dependent manner

486 (b). Representative images (c) and quantification (d) of trans-synaptically labeled tdTomato(+)

487 neurons in BST, MEA, and COApm at the specified anterior-posterior axis ( $n=4$; from 3

488 independent experiments). Scale bar $=500 \mu \mathrm{m}$. e-g, Virus encoding ChR2 (AAV 2 -hSyn-hChR2-

489 EYFP) was targeted to the AOB (e,f). Representative image of FOS expression in the COApm

490 upon photoactivation of the AOB, from $n=3$ animals $(\mathbf{g})$. Scale bar $=500 \mu \mathrm{m}$. $\mathbf{h}$, These data are

491 associated with Fig.1g. Number of FOS-expressing neurons in the vomeronasal pathway after

492 interaction with PBS- or LPS-females. $* * P<0.01, * * * P<0.001$ and $* * * * P<0.0001$ calculated by

493 unpaired two-tailed $t$-test (a), one-way ANOVA with Bonferroni's post-hoc test (d) and two-way

494 ANOVA with Sidak's post-hoc test (h). Graph indicates mean \pm s.e.m. p-values are described in

495 the supplementary statistical information file. 
a

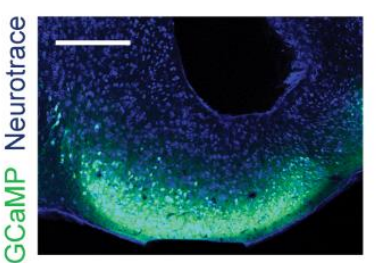

b \#1

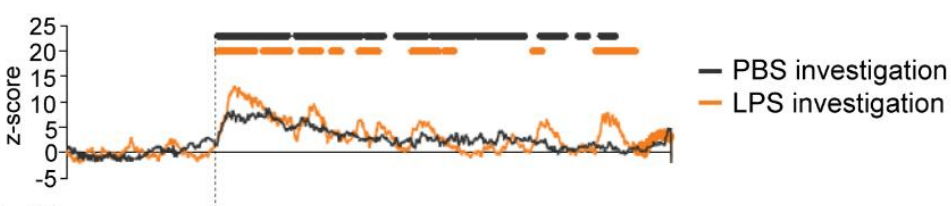

\#2

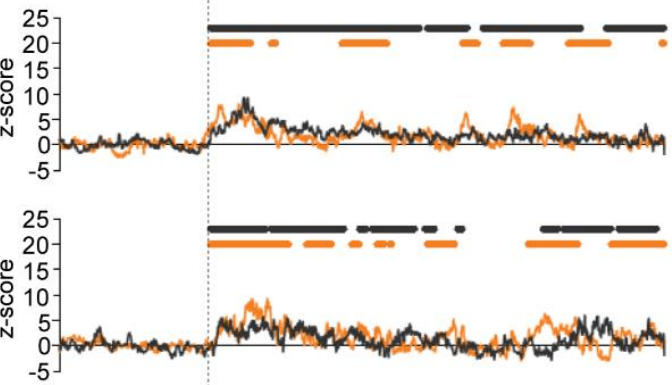

C
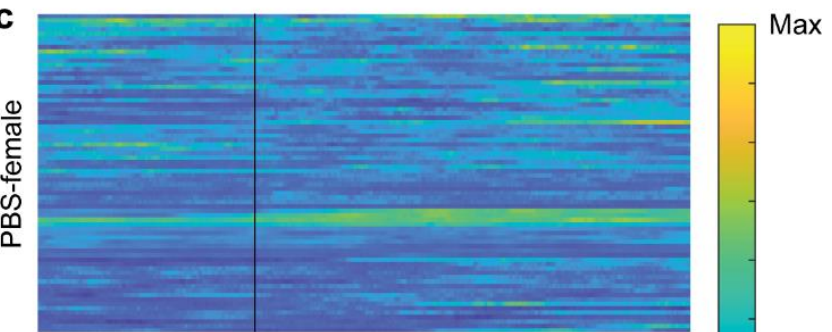

$\# 4$

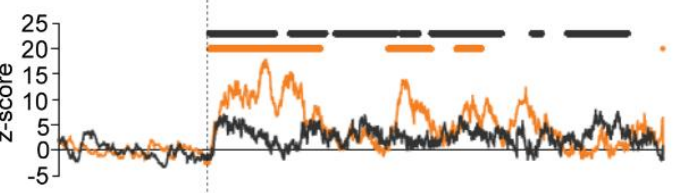

\#5
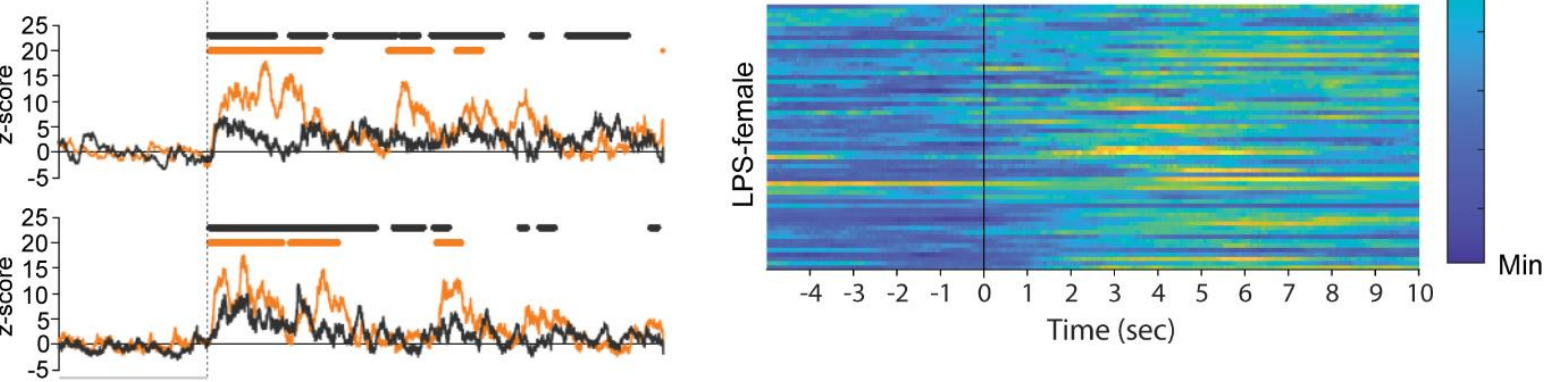

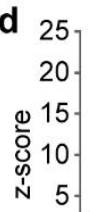

$(1 \mathrm{~min})$
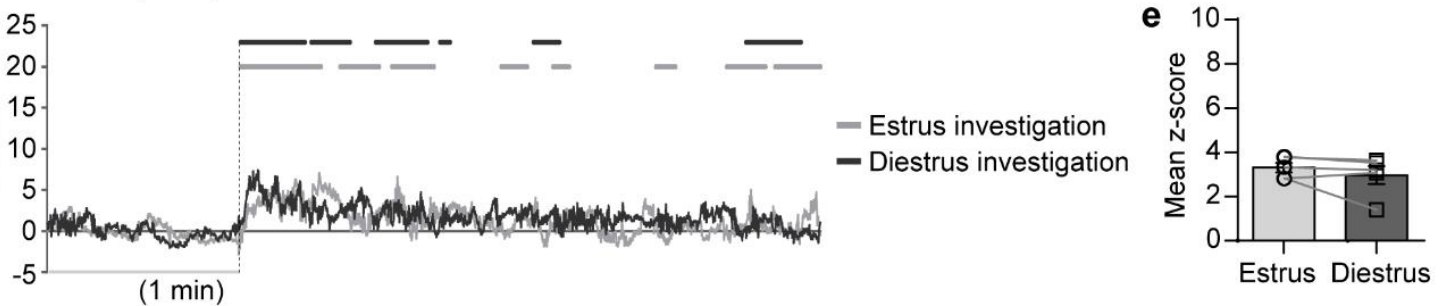

Estrus investigation

- Diestrus investigation
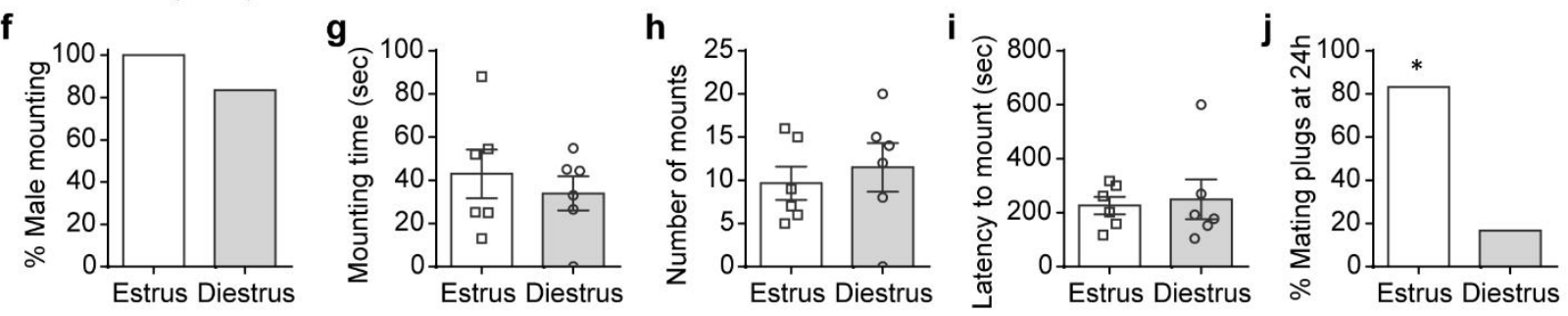

Extended Data Figure 3. Investigation of LPS-females induces neural activity in the 
500 GCaMP6s expression in the COApm (a). Individual traces of COApm bulk fluorescence signal

501 during interactions with a PBS- or LPS-female (b). Heatmap of normalized COApm responses to

502 PBS- or LPS-females. Each row represents a single investigation event. Investigation events

503 were pooled from 6 animals from 3 independent experiments. Time $=0$ indicates initiation of

504 investigation (c). Scale bar=300 $\mu$ m. d,e, Virus encoding GCaMP6s (AAV ${ }_{1}$-Syn-GCaMP6s) was

505 targeted to the COApm for fiber photometry recordings. Male mice were sequentially presented

506 with an estrus or diestrus female in counterbalanced sessions. Representative traces of COApm

507 bulk fluorescence signal (d) and the mean z-score of the fluorescence during direct investigation

508 of the estrus or diestrus female (e) (Estrus, $n=5$ and Diestrus, $n=5$; from 3 independent

509 experiments). $\mathbf{f - j}$, Male mounting behaviors towards an estrus or diestrus, healthy female.

510 Percent male mounting (f), mounting time (g), number of mounts (h), latency to mount (i), and

511 percent female partners with mating plugs (j) (Estrus, $n=6$ and Diestrus, $n=6$; from 2 independent

512 experiments). ${ }^{*} P<0.05$ calculated by Chi-Square Test of Independence (j). Graph indicates mean

$513 \pm$ s.e.m. p-values are described in the supplementary statistical information file. 
a

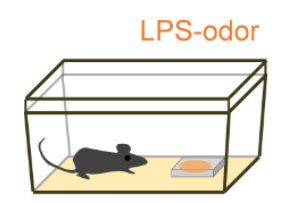

d

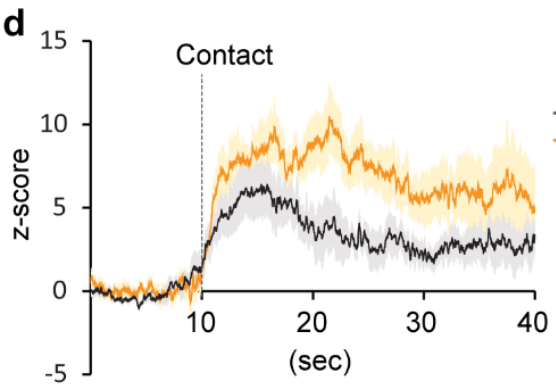

f
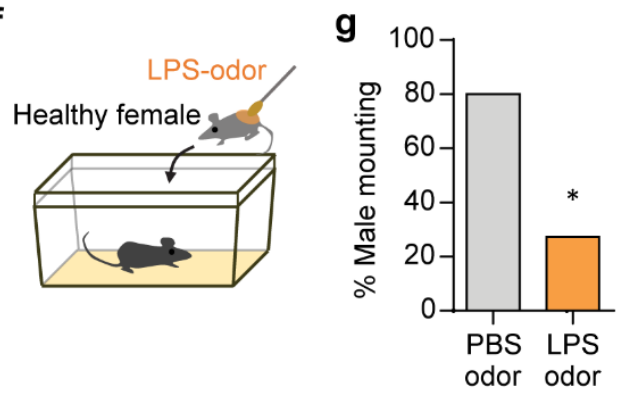
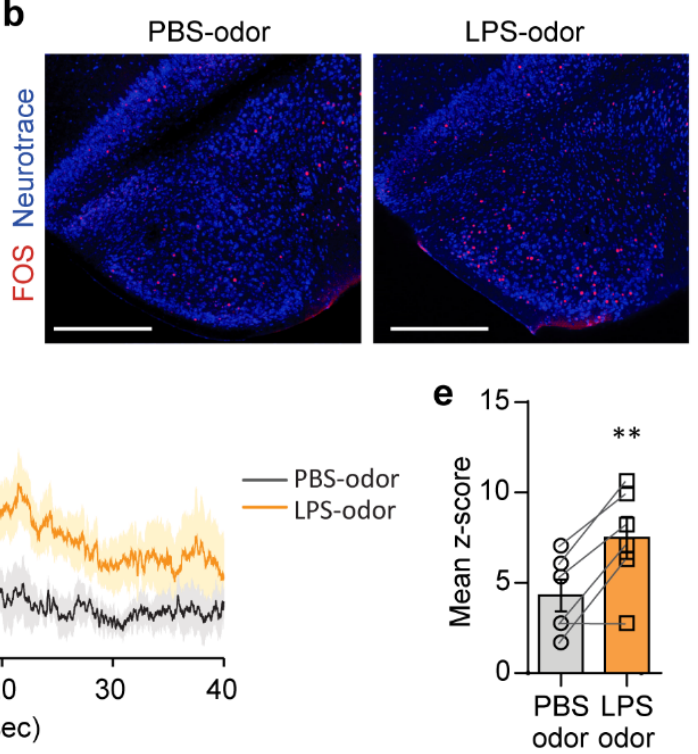

— PBS-odor
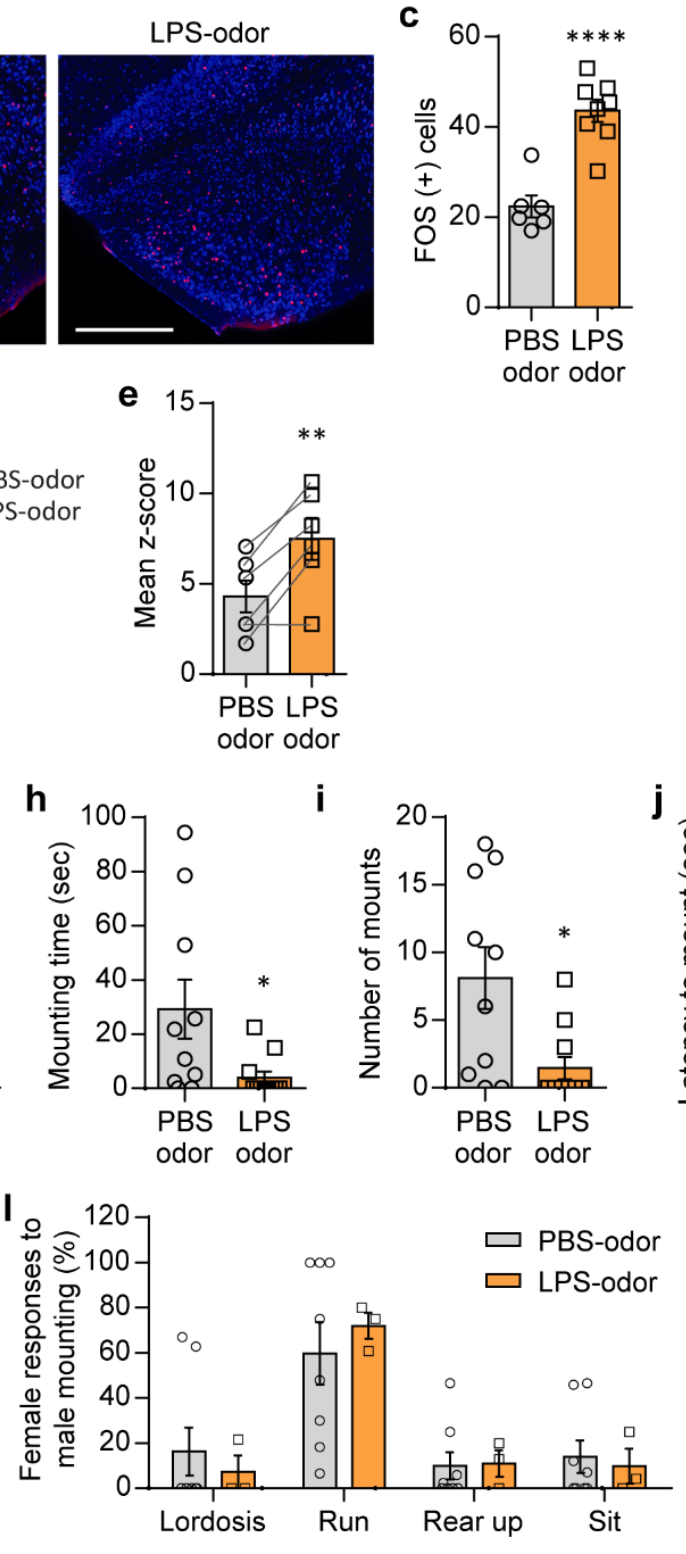
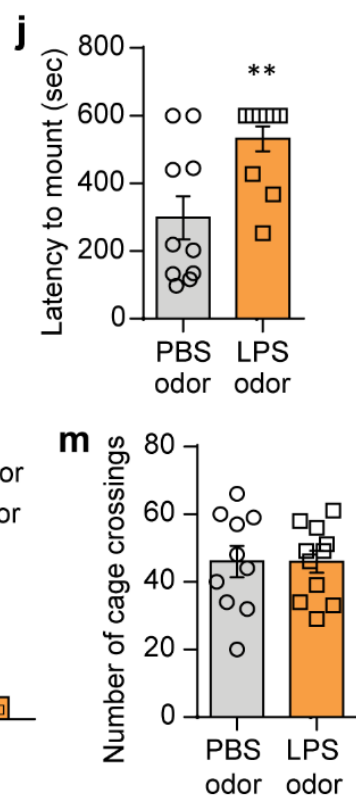

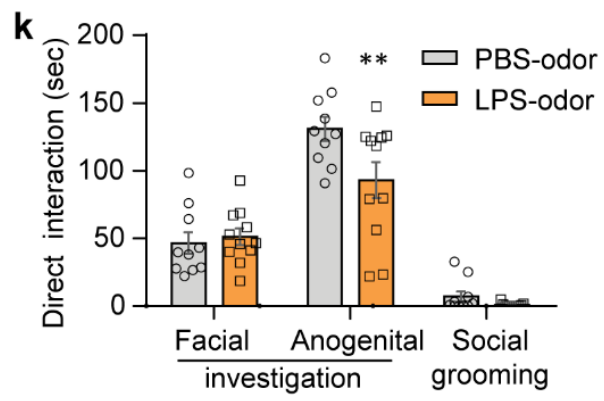

Extended Data Figure 4. LPS-odor suppresses male mating behaviors. a-c, Male mice were

517 presented with PBS- or LPS-odor (a). Representative images (b) and quantification (c) of FOS

518 expression in the COApm of males after exposure to PBS- or LPS-odor (PBS-odor, $n=6$ and

519 LPS-odor, $n=8$; from 2 independent experiments). Scale bar $=500 \mu \mathrm{m}$. d,e, Virus encoding

520 GCaMP6s (AAV 1 -Syn-GCaMP6s) was targeted to the COApm for fiber photometry recordings. 
521 Male mice were sequentially presented with a PBS- or LPS-odor in counterbalanced sessions.

522 Traces of COApm bulk fluorescence signal (solid line=average, shaded area=s.e.m.) $(\mathbf{d})$ and the

523 mean z-score of the fluorescence during the first $20 \mathrm{sec}$ of direct investigation of the odor (e)

524 ( $n=6$; from 3 independent experiments). f-m, Male mice were presented with a healthy female

525 painted with PBS- or LPS-odor (f). Percent male mounting (g), mounting time (h), number of

526 mounts (i), latency to mount (j), and duration of additional male behaviors while engaged in

527 direct interactions with the female (k). Percentage of female behaviors in response to males'

528 mounting attempts (l) and the number of cage crossings ( $\mathbf{m}$ ) (PBS-odor, $n=10$ and LPS-odor,

$529 n=11$; from 2 independent experiments). $* P<0.05$, $* * P<0.01$ and $* * * * P<0.0001$ calculated by

530 unpaired two-tailed $t$-test $(\mathbf{c}, \mathbf{h}-\mathbf{j})$, paired two-tailed $t$-test (e), Chi-Square Test of Independence

531 (g) and two-way ANOVA with Sidak's post-hoc test (k). Graphs indicate mean \pm s.e.m. p-values

532 are described in the supplementary statistical information file. 

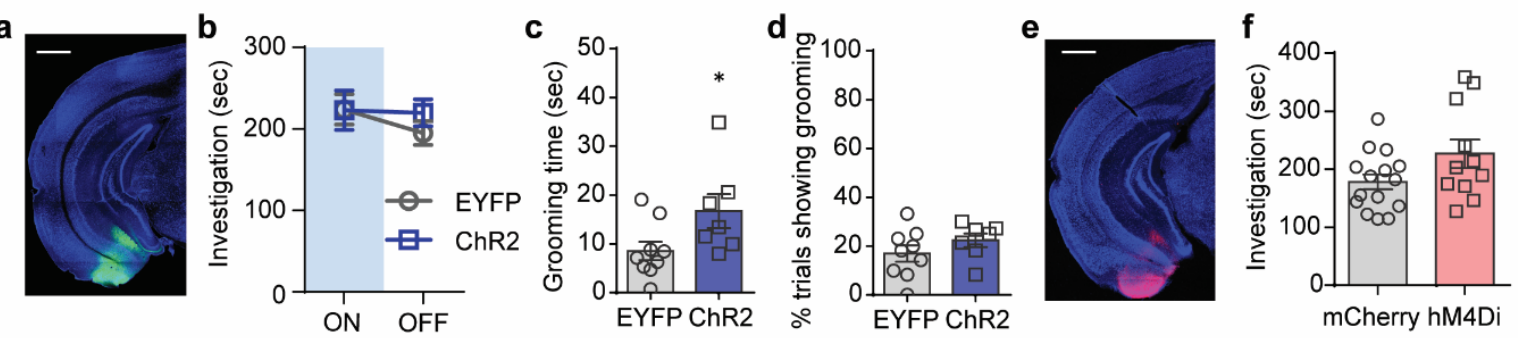

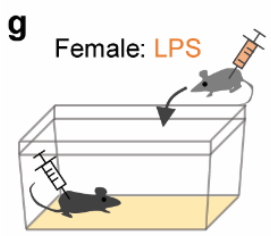

Saline or CNO

I

Healthy, estrus female

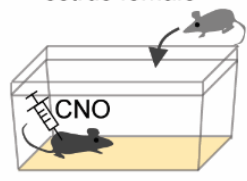

q Healthy, diestrus female

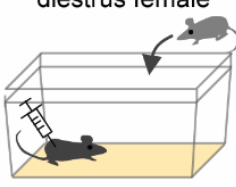

Saline or CNO
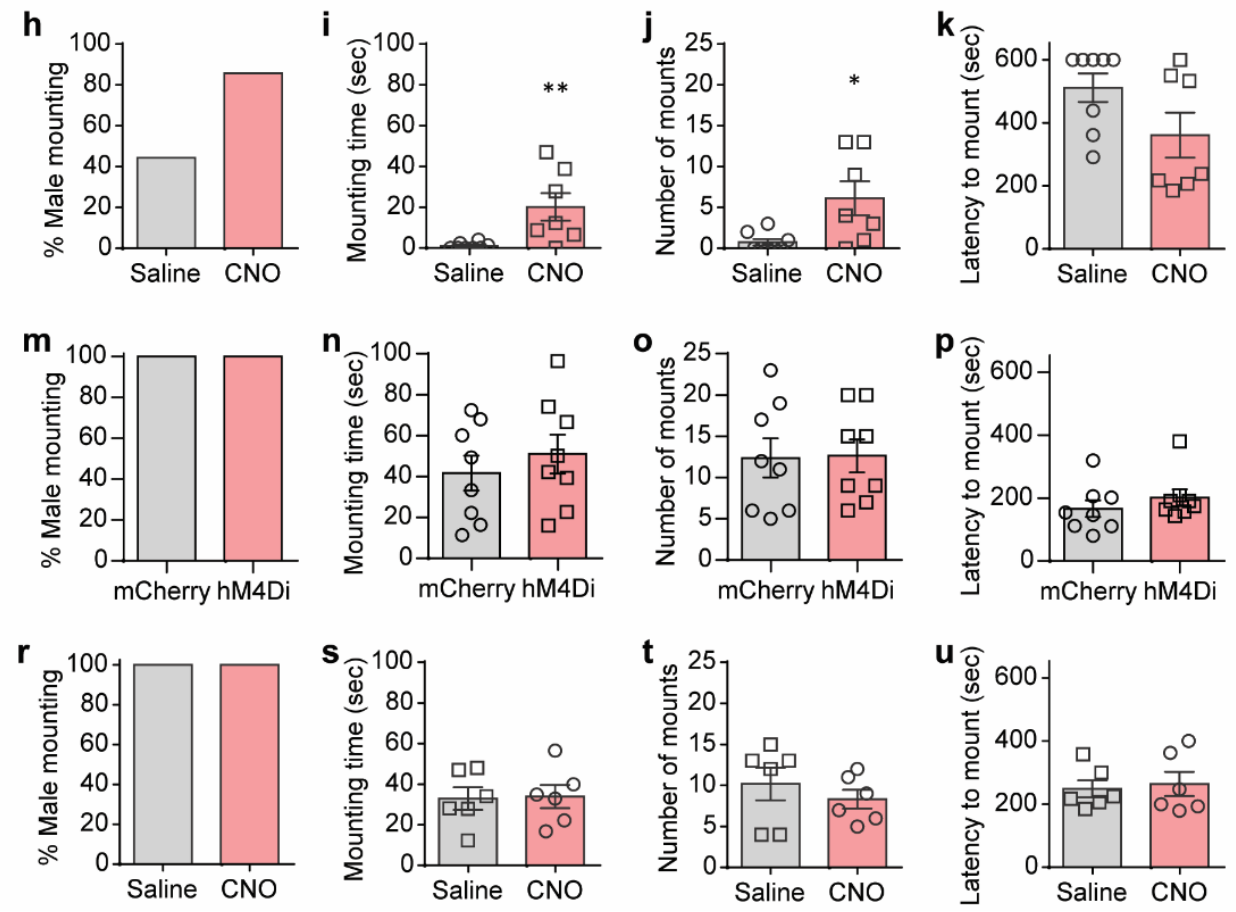

Extended Data Figure 5. COApm mediates suppression of mating behaviors towards

536 unhealthy females. a-d, These data are associated with Fig.2a-e. Representative image of ChR2

537 expression in the COApm (a). Total direct investigation time of healthy females in the presence

$538(\mathrm{ON})$ and absence (OFF) of COApm photoactivation (b). Duration of self grooming (c) and

539 percentage of photoactivation trials with self grooming (d). Scale bar=1mm. e,f, These data are

540 associated with Fig.2i-m. Representative image of hM4Di expression in the COApm (e) and

541 total direct investigation time of LPS-females (f). Scale bar=1 mm. g-k, Additional control

542 experiments for data in Fig.2i-m. Male mice expressing inhibitory DREADD (hM4Di, AAV $2^{-}$

543 hSyn-hM4D(Gi)-mCherry) in COApm were injected with either saline or CNO and tested for 
544 mounting behavior towards LPS-females (g). Percent male mounting (h), mounting time (i),

545 number of mounts ( $\mathbf{j}$ ), and latency to mount (k) (Saline, $n=8$ and CNO, $n=7$; from 2 independent

546 experiments). l-p, Male mice expressing mCherry ( $\mathrm{AAV}_{2}$-hSyn-mCherry) or inhibitory

547 DREADD (hM4Di, AAV 2 -hSyn-hM4D(Gi)-mCherry) in COApm were injected with CNO and

548 tested for mounting behavior towards untreated, healthy estrus females (I). Percent male

549 mounting (m), mounting time (n), number of mounts (o), and latency to mount ( $\mathbf{p})$ (mCherry,

$550 n=8$ and hM4Di, $n=8$; from 2 independent experiments). q-u, Male mice expressing inhibitory

551 DREADD (hM4Di, AAV 2 -hSyn-hM4D(Gi)-mCherry) in COApm were injected with saline or

$552 \mathrm{CNO}$ and tested for mounting behavior toward untreated, healthy diestrus females $(\mathbf{q})$. Percent

553 male mounting (r), mounting time (s), number of mounts (t)), and latency to mount (u) (Saline,

$554 n=6$ and CNO, $n=6$; from 2 independent experiments). $* P<0.05$, $* * P<0.01$ calculated by

555 unpaired two-tailed $t$-test $(\mathbf{c}, \mathbf{i}, \mathbf{j})$. Graphs indicate mean \pm s.e.m. $\mathbf{p}$-values are described in the

556 supplementary statistical information file. 

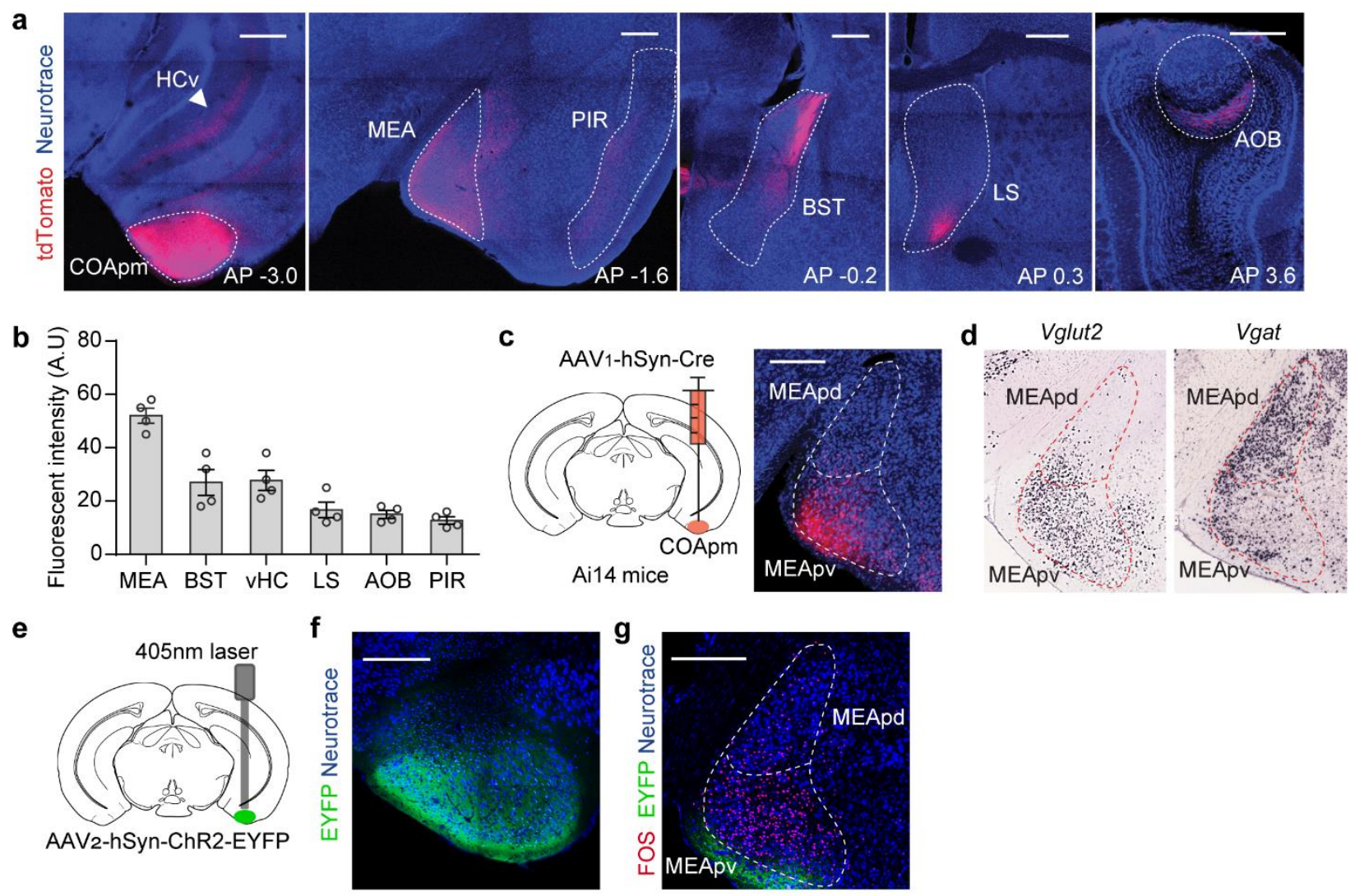

h

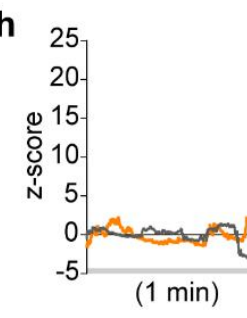

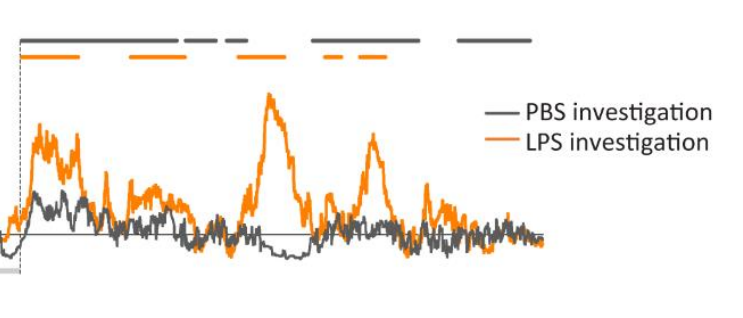

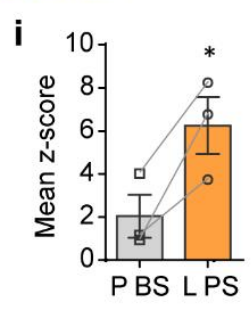

\section{Extended Data Figure 6. COApm neurons preferentially project to MEA-Vglut2(+)}

neurons. a-b, Virus encoding the anterograde tracer ( $\mathrm{AAV}_{2}$-hSyn-tdTomato) was targeted to the

561 COApm. Total fluorescence intensity was measured in sub-regions receiving COApm axonal

562 projections: MEA, BST, ventral hippocampus (HCv), lateral septum (LS), AOB and piriform

563 cortex $(\mathrm{PIR})(\mathrm{n}=4$ mice; from 2 independent experiments). Scale bar $=500 \mu \mathrm{m}$. c, Virus encoding

564 the anterograde trans-synaptic tracer ( $\left.\mathrm{AAV}_{1}-\mathrm{hSyn}-\mathrm{Cre}\right)$ was targeted to the COApm of Ai14

565 reporter mice that express tdTomato in a Cre-dependent manner. Representative image of MEA 
566 post-synaptic neurons at anterior-posterior (AP) $-1.7 \mathrm{~mm}$, from 3 independent experiments.

567 Scale bar $=300 \mu \mathrm{m}$. d, mRNA expression of Vglut2 and Vgat in MEA (Image credit: Allen

568 Institute). e-g, ChR2 ( $\mathrm{AAV}_{2}$-hSyn-ChR2-EYFP) was targeted to the COApm (e). Representative

569 images of ChR2 expression in COApm (f) and FOS expression in MEA upon photoactivation of

570 COApm (g) ( $n=3$; from 2 independent experiments). Scale bar $=300 \mu \mathrm{m}$. h,i, Virus encoding

571 GCaMP6s (AAV1-Syn-GCaMP6s) was targeted to the MEA of Vglut2-Cre mice for fiber

572 photometry recordings. Male mice were sequentially presented with a PBS- or LPS-female in

573 counterbalanced sessions. Representative traces of MEA-vGlut2(+) bulk fluorescence signal (h)

574 and the mean z-score of the fluorescence during direct investigation of the PBS- or LPS-female

575 (i) $(n=3$; from 2 independent experiments). $* P<0.05$ calculated by paired two-tailed $t$-test (i).

576 Graphs indicate mean \pm s.e.m. p-values are described in the supplementary statistical information 577 file. 


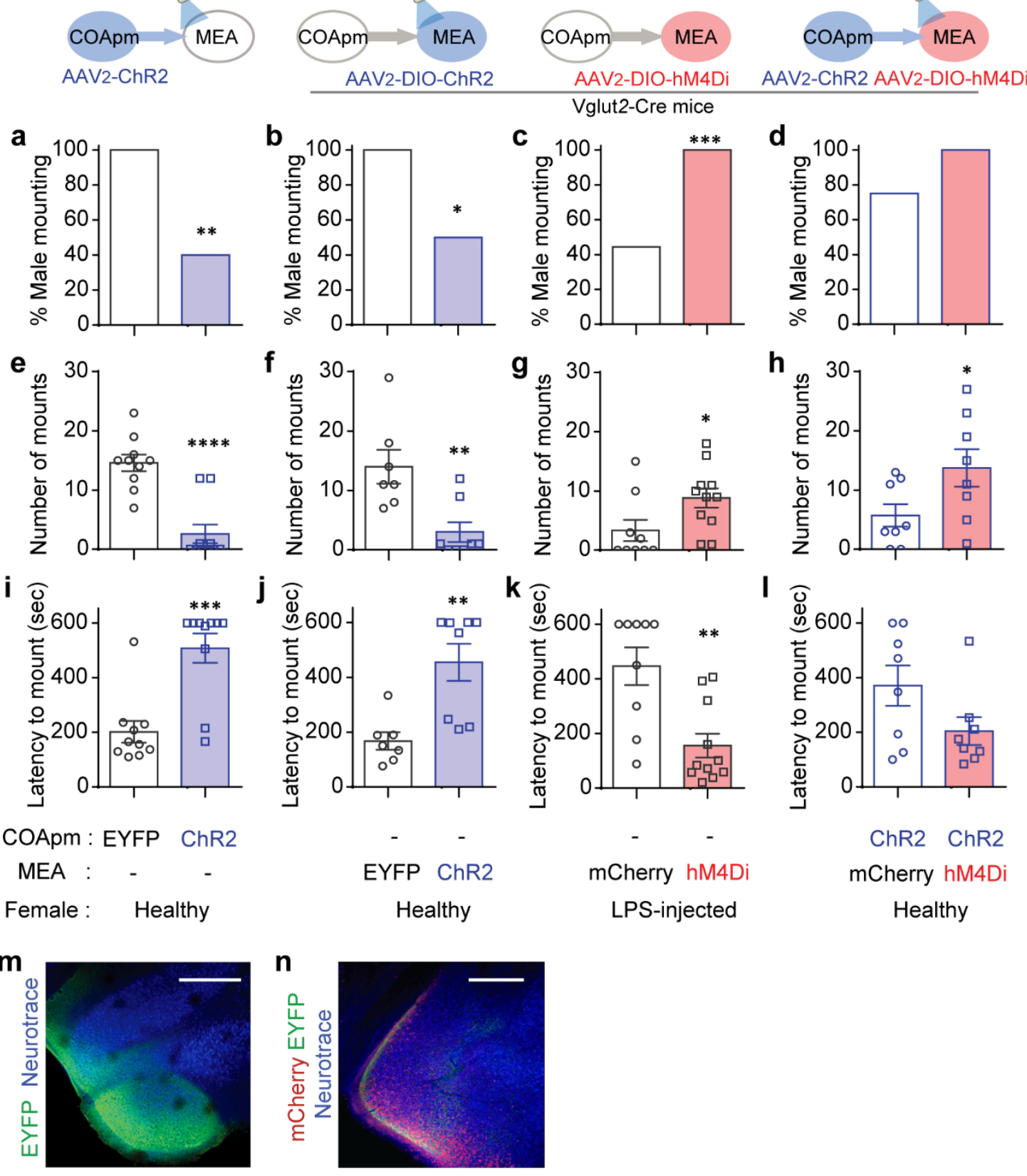

580 Extended Data Figure 7. COApm suppresses male mating behaviors by engaging MEA-

581 Vglut2(+) neurons. These data are associated with Fig.3f-i. Percent male mounting (a-d), 582 number of mounts (e-h), latency to mount (i-1). Representative images of ChR2 expression in 583 COApm (m) and hM4Di expression in MEA (n) of Vglut2-Cre mice with concurrent 584 photoactivation of COApm-MEA projections and hM4Di-inhibition of MEA-Vglut2(+) neurons. 
585 Scale bar $=500 \mu \mathrm{m} . * P<0.05, * * P<0.01$, $* * * P<0.001$ and $* * * * P<0.0001$ calculated by Chi-

586 Square Test of Independence (a-c) and unpaired two-tailed $t$-test (e-k). Graphs indicate mean \pm

587 s.e.m. p-values are described in the supplementary statistical information file.

588

589 


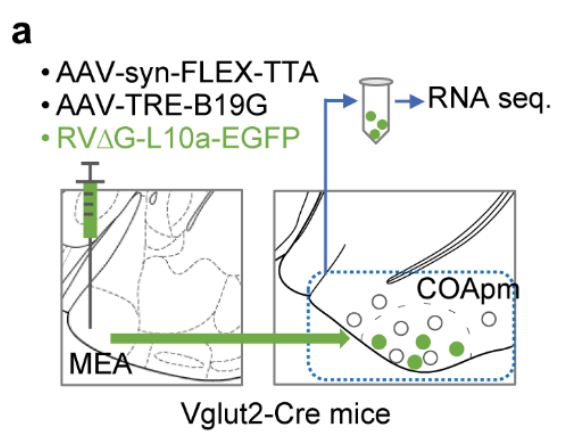

590

591

592

593

594

595

596

597

598

599

600

601 expressed genes that pass the threshold for the FDR are highlighted in red. c, Heatmap showing 602 COApm-differentially expressed genes that belong to the KEGG neuroactive ligand-receptor
603 interaction pathway. p-values are described in the supplementary statistical information file. 602 COApm-differentially expressed genes that belong to the KEGG neuroactive ligand-receptor
603 interaction pathway. p-values are described in the supplementary statistical information file.
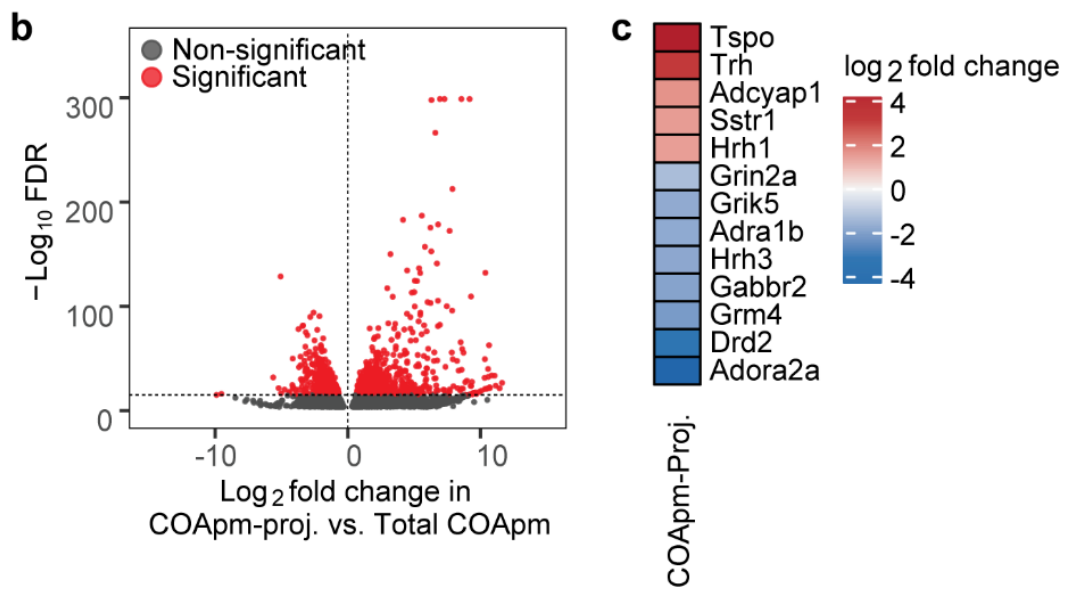

projecting to MEA-Vglut2(+) neurons. a, A combination of AAV ${ }_{1}$-syn-FLEX-TTA and AAVTRE-B19G, and RVAG-L10a-EGFP were sequentially injected into the MEA of Vglut2-Cre mice to label COApm neurons projecting to MEA-Vglut2(+) neurons (COApm-proj). COApm tissue was harvested and immediately used for TRAP analyses. Control mice were injected with AAV(PHP.eB)-Syn-L10a-EGFP via retro-orbital injection in order to label COApm neurons with L10a-EGFP independently of their efferent projections (Total COApm). b, Volcano plot showing $\log _{2}$-fold change plotted against - $\log _{10}$ FDR for the labeled COApm neurons projecting to the MEA-Vglut2(+) population (COApm-Proj.) compared to the Total COApm. Differentially 
a

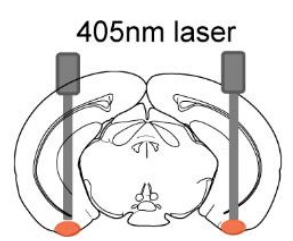

AAV2-Ef1a-DO-ChR2-mCh Trh-Cre mice
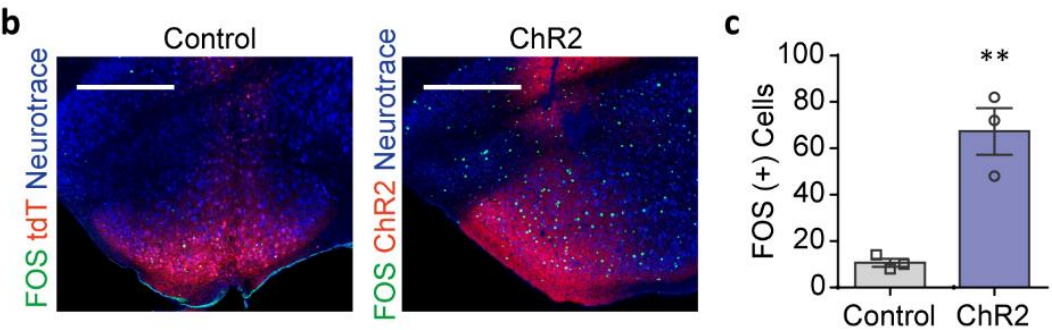

d
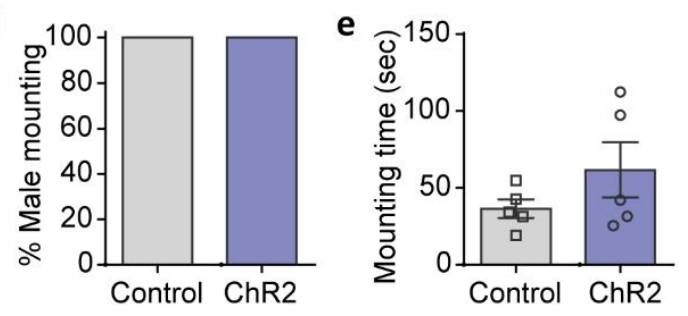

f

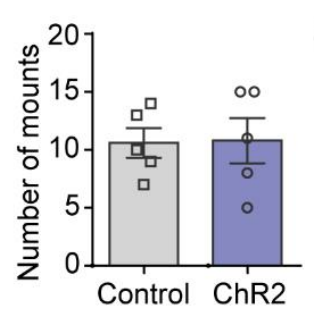

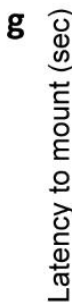

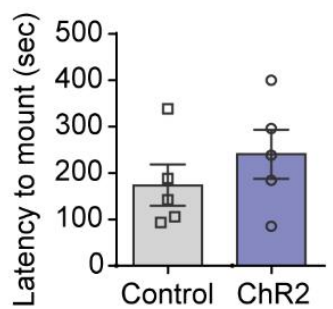

h
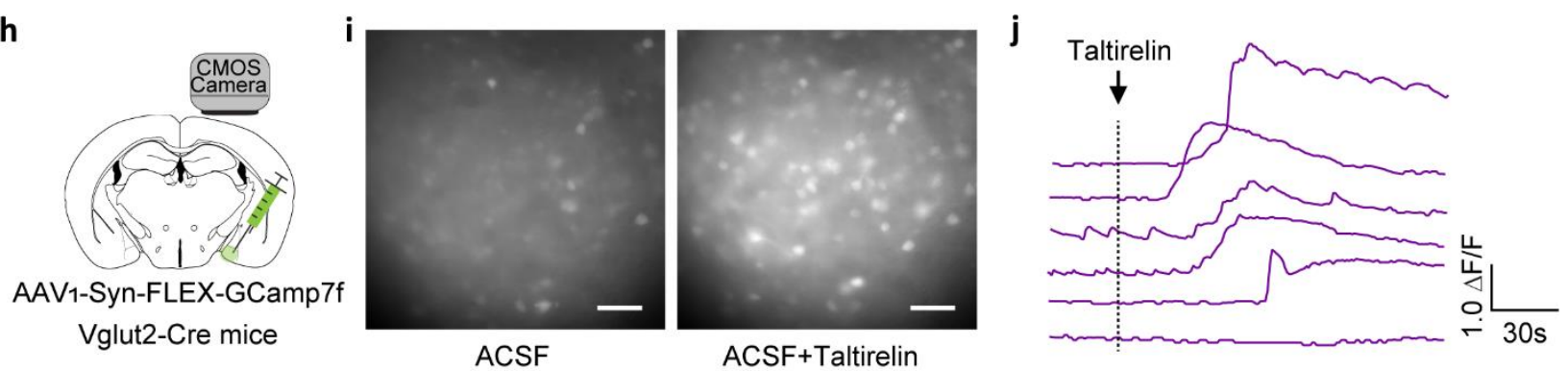

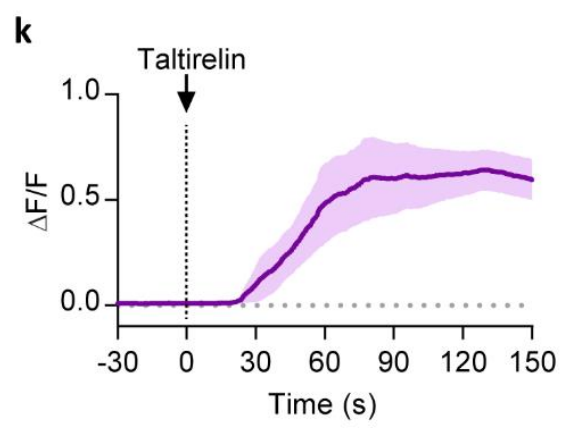

m

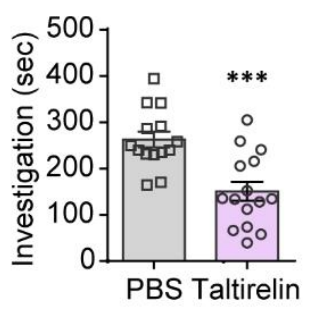

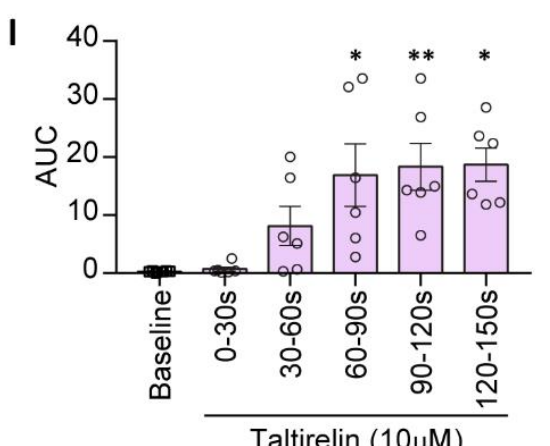

Taltirelin $(10 \mu \mathrm{M})$

607 towards unhealthy females. a-g, Trh-Cre male mice expressing tdTomato (AAV $1 / 2-E f 1$ a-DO- 
609 Cre(-) neurons were tested for mating behaviors towards healthy females with COApm

610 photoactivation (a). Representative images (b) and quantification (c) of FOS expression in

611 COApm. Percent male mounting (d), mounting time (e), number of mounts (f), and latency to

612 mount (g) with photoactivation of COApm-TRH(-) cells (Control, $n=5$ and ChR2, $n=5$; from 2

613 independent experiments). Scale bar $=500 \mu \mathrm{m}$. $\mathbf{h}-\mathbf{l}$, Calcium imaging of MEA brain slices from

614 Vglut2-Cre mice expressing GCaMP7f (AAV ${ }_{1}$-hSyn-FLEX-GCaMP7f) in MEA-Vglut2(+)

615 neurons upon taltirelin $(10 \mu \mathrm{M})$ application (h). Representative images of MEA slices before (-

$61615 \mathrm{~s})$ and after $(+35 \mathrm{~s})$ taltirelin application (i). Example traces of fluorescence signal from

617 individual neurons $(\mathbf{j})$ and the average of the fluorescence signal (solid line=average, shaded

618 area=s.e.m.) (k) upon taltirelin application. Area under the curve (AUC) of the average

619 fluorescence signal from individual MEA slices binned every $30 \mathrm{sec}(\mathbf{l})$ ( $\mathrm{n}=6$ slices, from 3

620 mice). Scale $=50 \mu \mathrm{m}$. $\mathbf{m}$, These data are associated with Fig.4k-o. Total duration of direct

621 investigation following microinjection of the TRH analog taltirelin into MEA. ${ }^{*} P<0.05$,

$622 * * P<0.01$ and $* * * P<0.001$ calculated by unpaired two-tailed $t$-test $(\mathbf{c}, \mathbf{m})$ and Friedman test with

623 Dunn's multiple comparisons test (I). Graphs indicate mean \pm s.e.m. p-values are described in the

624 supplementary statistical information file. 

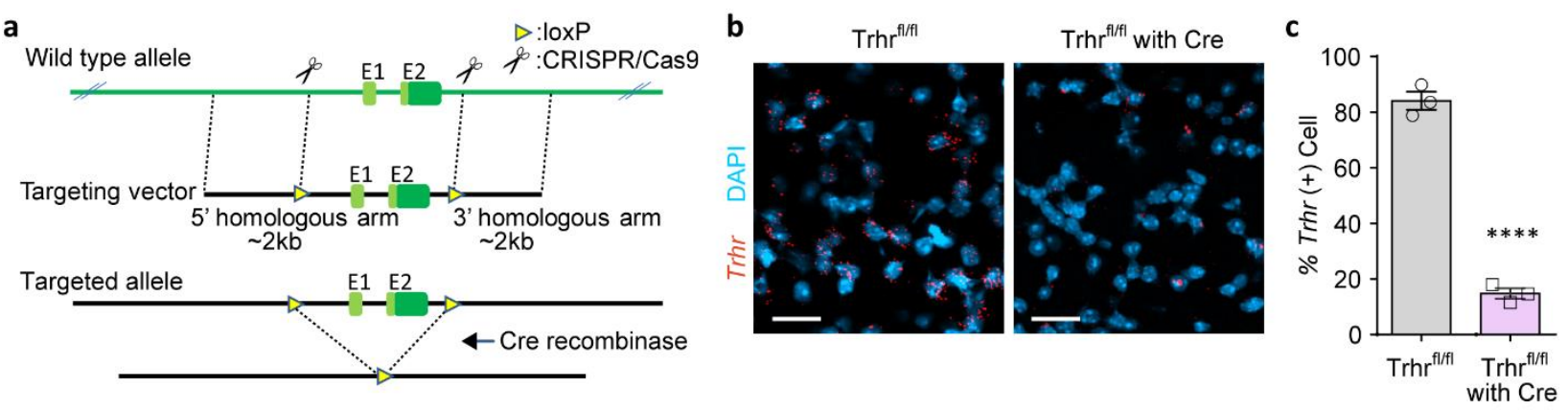

d
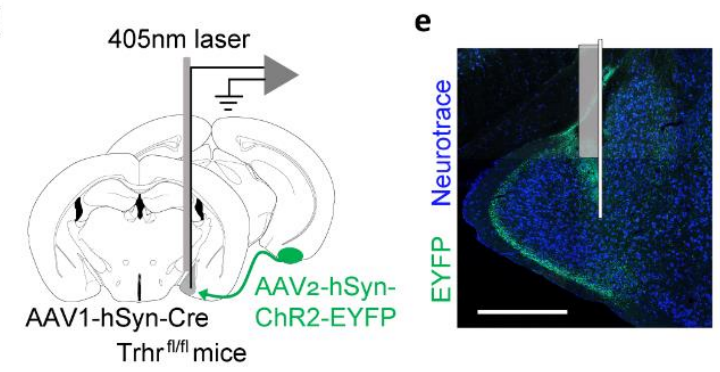

f

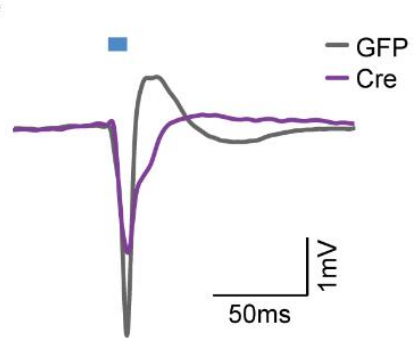

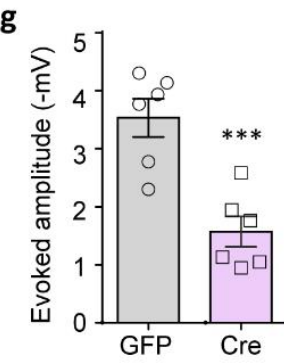

Extended Data Figure 10. Suppression of mating engages TRH-TRHR signaling in the

COApm-MEA projection. a, Schematic depicting the targeting construct used to generate the

Trhr conditional knock-out mouse line. b,c Representative images (AP -1.8 mm ) (b) and quantification (c) of Trhr mRNA expression in the MEApv of $\operatorname{Trhr}^{\mathrm{fl} / \mathrm{fl}}$ mice with or without Cre expression $\left(\mathrm{AAV}_{1}-\mathrm{hSyn}-\mathrm{Cre}\right)\left(\mathrm{Trhr}^{\mathrm{fl} / \mathrm{fl}}, \mathrm{n}=3\right.$ and $\mathrm{Trhr}^{\mathrm{fl} / \mathrm{fl}}$ with $\mathrm{Cre}, \mathrm{n}=3$; from 2 independent

632 experiments). Scale bar $=20 \mu \mathrm{m}$. d-g, In vivo recordings of MEA responses to COApm inputs in 633 Trhr conditional knock-out mice. Trhr ${ }^{\mathrm{fl} / f l}$ mice were injected with AAV 2 -hSyn-ChR2-EYFP in

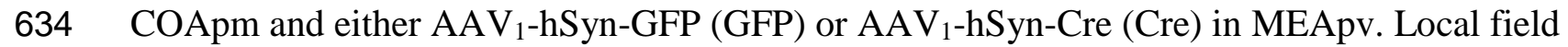
635 potentials evoked by a $10-\mathrm{msec}$ photoactivation were recorded from MEApv in anesthetized 636 mice using an optrode (d). Representative image of electrode localization (e). Representative 637 waveforms (f) and amplitudes (baseline-to-negative peak) of MEApv responses evoked by 638 photoactivation of COApm inputs (g) (GFP, $n=6$ and Cre, $n=6$; from 6 independent 639 experiments). Scale bar $=300 \mu \mathrm{m} .{ }^{*} * * P<0.001$ and $* * * * P<0.0001$ calculated by unpaired two- 
640 tailed $t$-test $(\mathbf{c}, \mathbf{g})$. Graphs indicate mean \pm s.e.m. p-values are described in the supplementary

641 statistical information file.

642 


\section{Methods}

\section{Animals}

645 All experiments were performed according to the Guide for the Care and Use of Laboratory

646 Animals and were approved by the National Institutes of Health and the Committee on Animal

647 Care at Massachusetts Institute of Technology. C57BL/6J (000664), Slc32a1(Vgat)-Cre

648 (028862), Slc17a6(Vglut2)-Cre (016963), and Ai14 (007914) were purchased from Jackson

649 Laboratories and inbred. Generation of Trh-IRES-Cre was described ${ }^{30}$. In order to develop a

650 conditional knockout mouse line that removes TRHR in a Cre-dependent manner, a targeting

651 vector with two loxP sites flanking common exons 1 and 2 was generated using the

652 CRISPR/Cas9 system (Biocytogen). For PCR genotyping, the following primers were used

653 (EGE-WFZ-023-5'loxp-F 5'-GTTCTGATGCCCAGATGGTCCTGTC-3', EGE-WFZ-023-

654 5'loxp-R 5'-TGCAGTCCCCAGGGCTTGAGATAAA-3' for WT 301bps/Floxed 378bps and

655 EGE-WFZ-023-3'loxP-F 5'-ACTGAGTGTGCTTTTAGAACCATGT-3', EGE-WFZ-023-

656 3'loxP-R 5'- TTTGGTTAATGGCCAGCTACACCTT-3' for WT 342bps/Floxed 415bps). Mice

657 were housed with $20-22^{\circ} \mathrm{C}, 40-60 \%$ humidity and a 12-hour light cycle and given ad libitum

658 access to food and water. All mice were males aged 2-5 months unless otherwise specified.

659

660 Stereotaxic surgery

661 Surgeries were carried out using aseptic techniques. Mice were anaesthetized using a mixture of

662 ketamine $(100 \mathrm{mg} / \mathrm{kg}$, intraperitoneal injection) and xylazine (10 mg/kg, intraperitoneal

663 injection). Mice were given preoperative slow-release buprenorphine $(1.0 \mathrm{mg} / \mathrm{kg}$, subcutaneous

664 injection). Injection coordinates from bregma in mm: COApm, $\pm 2.9 \mathrm{ML},-2.9 \mathrm{AP},-5.7 \mathrm{DV}$;

665 MEApv, $\pm 2.1 \mathrm{ML},-1.7$ AP, -5.6 DV; MEApd, $\pm 2.2 \mathrm{ML},-1.7$ AP, -5.4 DV; AOB, +1.0 ML, 
+4.25 AP, -1.8 DV with a 20-degree angle relative to AP. Injections were performed using a

667 pulled fine-glass capillary at $0.1 \mu 1 / \mathrm{min}, 1 \mu 1$ total for Cre-dependent viruses and $0.4 \mu 1$ otherwise.

668 For fiber photometry, AAV1-Syn-GCaMP6s or AAV1-Syn-FLEX-GCaMP7f (Penn Vector

669 Core $^{31}$ were delivered to COApm of wild-type mice or MEA of Vglut2-Cre mice, respectively,

670 in the right hemisphere with a $400-\mu \mathrm{m}$ diameter optic fiber implanted $300 \mu \mathrm{m}$ above the injection

671 targets. For experiments involving optical stimulation ${ }^{32}$, wild-type or Vgat/Vglut2/Trh-Cre mice

672 received bilateral stereotaxic injections of AAV2-hSyn-hChR2-EYFP (UNC Vector Core) or

673 AAV2-Ef1 $\alpha$-DIO-hChR2-EYFP (UNC Vector Core), respectively, with 300- $\mu$ m optic fibers

674 implanted $200 \mu \mathrm{m}$ above the injection targets. Control groups were injected with AAV2-hSyn-

675 EYFP or AAV2-Ef1 $\alpha$-DIO-EYFP, respectively, with optic fibers implanted similarly. For

676 photoactivation of COApm-MEA projections, wild-type mice received bilateral injections of

677 virus (AAV2-hSyn-hChR2-EYFP) to COApm with optic fibers implanted in MEA. For MEA

678 fiber photometry with COApm photoactivation, Vgat/Vglut2-Cre mice received unilateral

679 injections of virus encoding ChrimsonR (AAV2-hSyn-ChrimsonR-tdTomato (UNC)) to COApm

680 and virus encoding GCaMP6s (AAV1-CAG-Flex-GCaMP6s) to MEA. A 300- $\mu$ m optic fiber was

681 implanted in COApm for photoactivation and a 400- $\mu \mathrm{m}$ optic fiber was implanted in MEA for

682 fiber photometry. Optic fibers were prepared in-house as described previously ${ }^{33}$. For

683 photoactivation of AOB, wild-type mice received unilateral injection of AAV2-hSyn-hChR2-

684 EYFP to AOB. For photoactivation of TRH(-) cells, Trh-Cre mice received unilateral injection

685 of AAV1/2-Ef1a-DO-hChR2-mCherry (Addgene \#370820) to COApm. Control group was

686 injected with AAV1/2-Ef1a-DO-DIO-TdTomato-EGFP (Addgene \#37120). For DREADD

687 inhibition experiments ${ }^{34}$, wild-type or Vglut2-Cre mice received bilateral stereotaxic injections

688 of AAV2-hSyn-hM4D(Gi)-mCherry (Addgene) or AAV2-hSyn-DIO-hM4D(Gi)-mCherry 
(Addgene), respectively, either in COApm or MEA. AAV2-hSyn-mCherry or AAV2-hSyn-DIO-

690 mCherry were used for control groups, respectively. For administration of taltirelin into MEA,

691 guide cannulas (26 gauge, PlasticsOne) were implanted 500 $\mu \mathrm{m}$ above the MEA $( \pm 2.2 \mathrm{ML},-1.7$

692 AP, -5.1 DV). Guide cannulas were fitted with dummy cannulas to maintain cannula patency

693 after surgery. For genetic removal of Trhr in the MEA, wild-type or homozygous Trhr

694 conditional knock-out mice $\left(\operatorname{Trhr}^{\mathrm{fl} / \mathrm{fl}}\right)$ received bilateral injection of AAV 1 -hSyn-GFP or $\mathrm{AAV}_{1}$ -

695 Syn-Cre in the MEA. Removal of the vomeronasal organ was performed as previously

696 described ${ }^{35}$. Briefly, mice were anesthetized and the lower jaw was gently opened and fixed with

697 a homemade mouth holder. A midline incision was made in the soft palate and the underlying

698 bone was exposed. The rostral end of the VNO was exposed and bilaterally removed. The

699 resulting cavity was filled with a gel foam and the incision was closed with absorbable sutures.

700 All animals recovered for at least one week before behavioral testing.

701

702 Virus preparation

703 Chimeric $\mathrm{AAV}_{1} / \mathrm{AAV}_{2}{ }^{36}$ viruses were prepared with plasmids for pAAV-Ef1a-DO-hChR2-

704 mCherry (Addgene \#370820) and pAAV-Ef1a-DO-DIO-TdTomato-EGFP (Addgene \#37120).

705 Briefly, AAV plasmids, AAV1/AAV2 Rep/Cap, and adenovirus-helper plasmid (pFdelta6) were

706 transfected into HEK293FT cells and incubated for 3 days. Cells were lysed and rAAVs

707 harvested by centrifugation to remove cellular debris. Finally, rAAVs were purified using

708 heparin columns.

709

710 Tissue slice preparation and immunohistochemistry 
711 Mice were transcardially perfused with cold paraformaldehyde (PFA) (4\% in PBS). Brains were

712 kept in PFA overnight at $4{ }^{\circ} \mathrm{C}$ before vibratome sectioning (Leica VT1000s). Brains were cut at

$71350-\mu \mathrm{m}$ thickness for FOS immunohistochemistry. Brains were cut at $100-\mu \mathrm{m}$ thickness for all

714 other experiments. Sections were incubated in blocking solution ( $0.4 \%$ Triton X-100 and $2 \%$

715 goat serum in PBS) for $30 \mathrm{~min}$. Sections were then incubated in blocking solution containing

716 rabbit anti-FOS (1:500, Millipore, ABE457) primary antibody overnight at room temperature.

717 Sections were washed in wash buffer ( $0.4 \%$ Triton X-100 in PBS) three times and incubated in

718 blocking solution containing Alexa 488 Goat anti-rabbit (1:250, Life Technologies, A11034)

719 secondary antibodies and DAPI (1:5,000, D1306, Thermo Fisher) for two hours at room

720 temperature. Images of stained slices were acquired using confocal microscopes (LSM710 and

721 LSM900, Carl Zeiss) with a 10×, $20 \times$ or $40 \times$ objective lens.

722

\section{Anatomical tracing}

724 For anterograde tracing of COApm axonal projections, wild-type mice received unilateral

725 stereotaxic injections of virus ( $\mathrm{AAV}_{2}$-hSyn-tdTomato) into COApm. 4 weeks later, mice were

726 sacrificed and $100-\mu \mathrm{m}$ thick coronal brain slices were prepared. Images were taken from brain

727 regions expressing tdTomato every 300- $\mu$ m using a confocal microscope (LSM710, Carl Zeiss).

728 Using Zen software (Carl Zeiss), regions of interest were defined based on the Paxinos brain

729 atlas $^{37}$ and total fluorescence intensity in each region was calculated. For anterograde trans-

730 synaptic tracing, AAV1-hSyn-Cre $(0.5 \mu \mathrm{l})$ virus was injected into AOB in Ai14 reporter mice that

731 express tdTomato in a Cre-dependent manner. After two weeks, mice were sacrificed and

732 coronal brain sections were prepared of BST (AP: 0.2 -0.4), MEA (AP: -1.2 -2.1) and COApm

733 (-2.4 -3.2). Images were taken every 200- $\mu \mathrm{m}$ using a confocal microscope (LSM710, Carl Zeiss) 
734 and the number of tdTomato positive cells were manually counted. For anterograde trans-

735 synaptic tracing of COApm, the same histological and imaging procedures were used for MEA

$736(\sim \mathrm{AP}-1.7)$.

738 Quantification of FOS (+) cells

739 For FOS expression studies after interaction with healthy or sick females, subject male mice

740 were co-housed with a female for 3 days for habituation. The female was separated and

741 intraperitoneally injected with either PBS or LPS and 2 hours later returned to the male cage.

742 Males were sacrificed 90 min after a 2-min free interaction with the female and brains were

743 processed for immunohistochemical detection of FOS protein. All cells were counted bilaterally

744 from two coronal sections of each respective brain region, as defined by the Paxinos brain

745 atlas ${ }^{37}$. Regions quantified include: AOBmi: mitral layer of the accessory olfactory bulb, AOBgr:

746 granular layer of the accessory olfactory bulb, BST: bed nucleus of the stria terminalis, MEApd:

747 posterodorsal part of the medial amygdalar nucleus, MEApv: posteroventral part of the medial

748 amygdalar nucleus, COApm: posteromedial nucleus of the cortical amygdala. For FOS

749 expression studies after interaction with PBS- or LPS-odor, males were sacrificed 90 min after a

750 2-min exposure to urine and feces obtained from females intraperitoneally injected with PBS

751 (PBS-odor) or LPS (LPS-odor) and brains were processed for immunohistochemical detection of

752 FOS protein in the COApm. For FOS expression studies after photoactivation, males were

753 sacrificed 90 min after photoactivation of AOB or COApm and brains were processed for

754 immunohistochemical detection of FOS protein in the COApm.

755

756 Fiber photometry 
757 The fiber photometry system was built as previously described ${ }^{38}$. Briefly, blue light from a 473

$758 \mathrm{~nm}$ diode laser (Company, USA) was chopped at $197 \mathrm{~Hz}$ and reflected off of a dichroic mirror

759 (FF495, Semrock, NY, USA) and coupled into a $600 \mu \mathrm{m}$ optical fiber patch cord (0.48NA,

760 Thorlabs, BFH48-600) through a microscope objective (40 x 0.65 NA, Olympus). The patch

761 cord was coupled with a free optic fiber, which has the same light efficiency as the implanted

762 one, and light power at the fiber tip was set to $20 \mu \mathrm{W}$. The patch cord was connected to an

763 implanted $400 \mu \mathrm{m}$ optical fiber via a ceramic sleeve. GCaMP6s fluorescence was collected

764 through a bandpass filter (FF01-520/35) into a photodetector (2151, NewFocus). The signal was

765 passed through a lock-in amplifier (Model SR810, Stanford Research Systems, CA, USA) and

766 digitized and collected with a LabVIEW DAQ (100Hz sampling frequency, LabVIEW), and

767 recorded by custom LabVIEW software. Animal behavior was recorded using EthoVision XT

768 tracking system (Noldus, Wageningen, Netherlands) and time-stamped with photometry data

769 using the LabVIEW TTL pulse. Male mice expressing GCaMP in COApm or MEA were single

770 housed and habituated with fiber coupling in their home cage placed in the behavior chamber for

$77130 \mathrm{~min}$. During this habituation, the fluorescence signal level was checked and the mice showing

772 basal fluorescence level between 3-5 amplitudes were used for further experiments to minimize

773 the signal intensity variation between animals, which might be caused by variable GCaMP

774 expression levels and optic fiber placements. A stimulus mouse (PBS- or LPS-females in estrus;

775 estrus or diestrus healthy females; healthy females painted with PBS- or LPS-odor) or stimulus

776 odor (PBS- or LPS-odor) were presented for $10 \mathrm{~min}$ in sequential and counterbalanced sessions

777 with an 1h-interval in between the sessions. Urine and feces from PBS- or LPS-females served as

778 PBS- or LPS-odor. The raw fluorescence signal was normalized to the mean of the 1-min

779 baseline prior to the first contact for z-score calculation. The mean z-score of fluorescence 
780 during direct investigation was calculated from the first 3-min periods. For the generation of the

781 heat map, z-scored fluorescence signals were further min-max normalized.

\section{Behavioral analyses}

784 All behavior experiments were performed between three hours before and after light offset time

785 (light offset: 7:00pm). Mice were transferred to the testing area at least three hours before the

786 initiation of experiments. Tracking of mouse behavior was done using the EthoVision XT

787 (Noldus, Wageningen, Netherlands) tracking system.

788 Mating assays. Naive C57BL/6J females were used. All females were in estrus, unless

789 otherwise indicated to be in diestrus. Estrus phase was checked by vaginal smear on the testing 790 day $^{39}$. Male mice were first screened for mounting behavior. After 30-min of habituation, they

791 were presented with a female in its home cage for $5 \mathrm{~min}$. Only the males that mounted the female

792 within the first 5 min period were used for experimentation. Males that successfully mounted

793 were then single-housed for a minimum of three days prior to the behavior test. For testing, the

794 home-cage of a male mouse was placed in a behavioral chamber with recording capabilities with

795 a side view camera. After a 10 min acclimation in the behavioral chamber, an intruder female

796 was placed into the male's home cage for $10 \mathrm{~min}$ before being removed. Only for the

797 experiments described in Extended Data Fig. 1a-c, two females were simultaneously introduced.

798 Intruder females were either untreated, or intraperitoneally injected with PBS (PBS-female) or

799 LPS (LPS-female, $0.5 \mathrm{mg} / \mathrm{kg}$ ) 2 hours before testing, or painted with urine and feces from PBS-

800 females (PBS-odor) or LPS-females (LPS-odor). Male mounting attempts (percent males

801 engaged in mounting, mounting time, number of mounts, and latency to the first mounting

802 attempt), investigation (sniffing of facial or anogenital regions), and social or self-grooming were 
manually scored. Four different categories of female behaviors (Run: the display of an active

804 escaping behavior from males' mounting attempts; Rear up: the display of a defensive posture by

805 facing up against the male during mounting attempts; Sit: the display of a passive, non-receptive

806 response by sitting down and not exposing the genital region during males' mounting attempts;

807 Lordosis: the display of receptive responses, including the canonical lordosis posture with the

808 curved spine and a standing posture with four limbs without the curved spine, that allow for

809 males mounting) displayed upon males' mounting attempts were manually scored. For the

810 behavioral raster plots shown in Extended Data Figure 1i, female behaviors during the rest of the

811 interactions were also analyzed and these include exploration of the cage, which is a novel

812 environment to the females (e.g. sniffing and digging), and a sitting posture. The presence of

813 mating plugs in females was determined 24 hours following the mating assay to estrus versus

814 diestrus females, in which the females stayed with the males after the 10-min test.

815 Mating assays with optogenetic activation. ChR2, EYFP or tdTomato was virally targeted to

816 COApm, COApm-TRH(+), COApm-TRH(-) or MEA-Vglut2(+) and optic fibers were bilaterally

817 implanted. Mice were habituated for fiber coupling a day before testing. On the testing day, an

818 optic cable was connected to the optic fiber to deliver $405 \mathrm{~nm}$ laser stimulation. After a 10 -min

819 acclimation, a female was placed in the subject's home cage. Mice behavior was monitored

820 through a side-view camera and a 10 -sec photoactivation $(2 \mathrm{~mW}, 20 \mathrm{~Hz}$ square wave, $10 \mathrm{~ms}$ pulse

821 width) was manually triggered at each instance of contact investigation.

822 Mating assays with DREADD inhibition. Male mice expressing hM4Di or mCherry in

823 COApm or MEA-Vglut2(+) were habituated for intraperitoneal injection a day before testing in

824 the testing room. On the testing day, Clozapine N-Oxide $(1 \mathrm{mg} / \mathrm{kg})$ was diluted in sterile saline

825 and intraperitoneally administered to male mice $40 \mathrm{~min}$ prior to testing. 
827 DREADD inhibition of MEA-Vglut2(+) neurons. Experimental group mice were Vglut2-Cre 828 male mice bilaterally expressing ChR2 in COApm and hM4Di in MEA-Vglut2(+) neurons with 829 optic fibers implanted in MEA. Control group mice were Vglut2-Cre mice expressing ChR2 in 830 COApm and mCherry in MEA-Vglut2(+) neurons. Clozapine N-Oxide $(1 \mathrm{mg} / \mathrm{kg})$ was diluted in 831 sterile saline and administered to male mice 40 min prior to testing. Photoactivation of COApm832 MEA projections was manually triggered at each instance of contact investigation.

833 Mating assays with Taltirelin injection to MEA. Wild-type male mice with guide cannulas 834 implanted in MEA were habituated for injection cannula insertion a day before testing. On the 835 testing day, mice were briefly anesthetized using isoflurane, and either vehicle or Taltirelin (10 $836 \mathrm{ng} /$ side in $0.5 \mu \mathrm{l}$ at a rate of $180 \mathrm{nl} / \mathrm{min}, 7956-\mathrm{ML} / \mathrm{CF}, \mathrm{R} \& \mathrm{D})$ was administered bilaterally into 837 the MEA through 500- $\mu$ m projecting injector tips (PlasticsOne). Mice were returned to the home 838 cage and placed in a behavioral chamber. 20 min after administration of vehicle or taltirelin, 839 mice were assayed for mating behavior. Cannula placements were histologically verified.

840 Feeding assay. Male mice expressing ChR2 in COApm were single-housed and food-deprived

841 for 12 hours. Mice were then connected to optic fibers and placed back in their home cage.

842 Following 10 min, a single pre-weighed food pellet was placed in the center of their home cage.

843 The amount of time spent eating was measured over a ten-minute period, and the pellet was

844 removed and re-weighed to calculate the amount of food eaten. Photoactivation of COApm (2

$845 \mathrm{~mW}, 20 \mathrm{~Hz}$ square wave, $20 \%$ duty cycle) occurred throughout the 10-min testing period.

846 Three-chamber social approach assay. Single-housed male mice expressing ChR2 in COApm

847 were assayed for sociability using a three-chamber social approach assay. The arena was

848 constructed of white acrylic $(50 \mathrm{~cm} \times 35 \mathrm{~cm} \times 30 \mathrm{~cm})$. Wire cups (Spectrum Diversified) were 
849 placed in the back left and right corner of the arena beneath water-filled 1-L bottles (Nalgene).

850 On day 0 , mice were habituated to the arena for $20 \mathrm{~min}$ and returned to their home cage. On day

851 1, mice were connected to optic fibers and placed in the center of the arena and allowed to freely

852 explore. Following 10-min of habituation, mice were confined to the center of the arena. An

853 inanimate object (rubber stopper) or a male conspecific were placed beneath the wire cups.

854 Placement of the inanimate object and social target were alternated. Mice were then allowed to

855 freely explore the arena for $10 \mathrm{~min}$. During this period, photoactivation of COApm ( $2 \mathrm{~mW}, 20$

$856 \mathrm{~Hz}$ square wave, $20 \%$ duty cycle) was automatically triggered during interaction with either the

857 inanimate object or social target. Interaction time was defined as time spent in the areas

858 circumscribing the wire cups $(<2 \mathrm{~cm})$. Sociability was defined as interaction time with the social

859 target divided by total interaction time and expressed as a percentage.

860 Three-chamber social preference assay. Single-housed wild-type mice were used for the

861 behavioral assay as described above ("Three-chamber social approach assay"), except the target

862 objects were PBS- and LPS-females.

863 Real time place preference. Male mice expressing ChR2 in COApm were assayed for

864 preference of COApm photoactivation in a real time place preference (RTPP) assay. The arena

865 consisted of two chambers constructed of white acrylic $(30 \mathrm{~cm}$ x $30 \mathrm{~cm})$. Mice were connected to

866 optic fibers and placed in the left chamber. Photoactivation of COApm ( $2 \mathrm{~mW}, 20 \mathrm{~Hz}$ square

867 wave, $20 \%$ duty cycle) was triggered whenever the mouse entered the right chamber. Stimulation

868 preference was defined as the time spent in the chamber paired with photoactivation divided by

869 total trial duration (10 min) and displayed as a percentage.

870

\section{$871 \quad$ In situ hybridization}


872 Fluorescence in situ hybridization (FISH) was conducted with ACDBio RNAscope Multiplex

873 Fluorescence Assay. Following probes were used: C1: Trhr (Mm-Trhr: 443771), C2: Vglut2

874 (Mm-Slc17a6-C2: 319171-C2), C3: Vgat (Mm-Slc32a1-C3: 319191-C3); C1: Trh (Mm-Trh:

875 436811). Brain tissues were obtained from age-matched 8-10 week-old sexually naïve male

$876 \mathrm{C} 57 \mathrm{BL} / 6 \mathrm{~J}$ mice and immediately fresh-frozen in optimal cutting temperature (OCT) medium on

877 dry ice. Twenty-micrometer sections were obtained using a cryostat (Leica, Germany) and stored

878 at $-80^{\circ} \mathrm{C}$. RNAscope procedures were performed according to the user manual provided by

879 ACDBio with modifications specified as following. Briefly, sections were fixed with $4 \%$ chilled

880 paraformaldehyde (PFA) for $15 \mathrm{~min}$ and washed in $1 \times$ phosphate-buffered saline (PBS) twice

881 for 2 min each. Sections were then dehydrated consecutively in 50\%, 75\%, 100\%, and 100\%

882 ethanol for $5 \mathrm{~min}$ each. Tissue was digested with Protease IV for $13 \mathrm{~min}$ at room temperature and 883 washed twice in 1 $\times$ PBS with agitation. Vglut2 and Vgat probes were diluted 1:50 in Trhr probe

884 solution and pipetted onto each slide. Probe hybridization was conducted for $2 \mathrm{~h}$ and $30 \mathrm{~min}$ at $88540^{\circ} \mathrm{C}$ and slides were then washed in $1 \times$ wash buffer twice for 4 min each. Sections were then 886 incubated at $40^{\circ} \mathrm{C}$ in Amp1 for $35 \mathrm{~min}$, Amp2 for $17 \mathrm{~min}$, Amp3 for $35 \mathrm{~min}$, and Amp4 for $88717 \mathrm{~min}$, with two $1 \times$ Wash Buffer rinses between each incubation. Sections were finally treated 888 with DAPI for $30 \mathrm{sec}$, mounted with $\mathrm{CC} /$ Mount and stored at $4{ }^{\circ} \mathrm{C}$. Images were acquired using 889 Zeiss LSM 710 and LSM900 confocal microscopes (x40 objective, 1.2 NA) and a Zeiss Axio 890 Imager.Z2 microscope (x10 objective, $0.45 \mathrm{NA}$ ). Imaging settings were established during the 891 first acquisition and not modified afterwards. Target genes (Trh, Trhr, Vgat, and Vlut2) and 892 DAPI expression were quantified using QuPath ${ }^{40}$. For Trh quantification, cells were divided into 893 the following categories based on level of Trh expression: low $=1-3$ puncta, medium $=4-9$ 894 puncta, high $=10-15$ puncta, highest $=>15$ puncta. For co-expression of Vglut2, Vgat and Trhr, 
895 cells were classified as positive if the level of target expression was $>3$ puncta. Percentage of co896 expression is calculated as the number of Vglut2 and Trhr or Vgat and Trhr double positive cells

897 divided by the total number of Trhr positive cells. For validation of conditional knock-out of

898 Trhr in the MEA, Trhr probe was used on brain tissues from Trhr ${ }^{\mathrm{fl} / f l}$ animals, which

899 received stereotaxic injections of $\mathrm{AAV}_{1}$-Syn-Cre in MEApv. Trhr ${ }^{\mathrm{fl} / \mathrm{fl}}$ animals without virus

900 injection served as a control. To quantify the percentage of MEApv neurons expressing Trhr, 901 cells were classified as Trhr-positive if the level of target expression was $>1$ puncta.

902

903 Calcium imaging of MEA brain slices

904 Slice preparation. Virus (AAV ${ }_{1}$-Syn-FLEX-GCaMP7f) was bilaterally delivered to MEA of 905 vGlut2-Cre mice. 4-8 weeks after the virus injection, mice were anesthetized with isoflurane.

906 Before decapitation, intracardiac perfusion was performed with an ice-cold cutting solution 907 containing (in mM): $96 \mathrm{~N}$-Methyl-D-glucamine (NMDG), $2.5 \mathrm{KCl}, 10 \mathrm{MgSO} 4,0.5 \mathrm{CaCl} 2,1.25$

$908 \mathrm{NaH} 2 \mathrm{PO} 4,25 \mathrm{NaHCO} 3,25$ glucose, 17.5 HEPES, $5 \mathrm{Na}$ ascorbate, 2 thiourea, 3 Na pyruvate, 12

909 L-acetyl-cysteine, perfused with $95 \% \mathrm{O}_{2}$ and $5 \% \mathrm{CO}_{2}(\mathrm{pH}$ 7.3-7.4 adjusted by $\mathrm{HCl}$ after

910 bubbling). Coronal slices of the MEA (300 $\mu \mathrm{m}$ thick) were obtained by a vibratome (VT1200s,

911 Leica) in the ice-cold cutting solution. The slices were immediately transferred to the same

912 cutting solution at $34{ }^{\circ} \mathrm{C}$ for $10 \mathrm{~min}$, and then transferred to slice holding solution at room

913 temperature containing (in mM): $94 \mathrm{NaCl}, 2.5 \mathrm{KCl}, 2 \mathrm{MgSO} 4,2 \mathrm{CaCl} 2,1.25 \mathrm{NaH} 2 \mathrm{PO} 4,25$

914 NaHCO3, 25 glucose, 14 HEPES, $5 \mathrm{Na}$ ascorbate, 2 thiourea, 3 Na pyruvate, 12.3 L-acetyl-

915 cysteine, perfused with $95 \% \mathrm{O}_{2}$ and $5 \% \mathrm{CO}_{2}(\mathrm{pH}$ 7.3-7.4 adjusted by $\mathrm{NaOH}$ after bubbling). The

916 slices were recovered in the holding solution for a minimum of 1.5 hours. All experiments were

917 done within 6 hours from the start of the recovery. 
918 Image acquisition and data analysis. Slices were transferred from the holding solution to an

919 immersion recording chamber perfused with artificial cerebrospinal fluid (ACSF) containing (in

$920 \mathrm{mM}): 126 \mathrm{NaCl}, 4 \mathrm{KCl}, 1 \mathrm{MgCl}_{2}, 1 \mathrm{CaCl}_{2}, 1 \mathrm{NaH}_{2} \mathrm{PO}_{4}, 26 \mathrm{NaHCO}_{3}, 20$ glucose, perfused with

$92195 \% \mathrm{O}_{2}$ and $5 \% \mathrm{CO}_{2}$. Flow of ACSF into the recording chamber was at a rate of $3 \mathrm{~mL} / \mathrm{min}$ and

922 its temperature was maintained at $33-34{ }^{\circ} \mathrm{C}$ by a temperature controller (TC-324C, Warner

923 Instruments). An upright microscope (SliceScope Pro 2000, Scientifica) with a 40X water

924 immersion objective lens (LUMPLFLN40XW, Olympus), $490 \mathrm{~nm}$ LED illumination (pE-100,

925 CoolLED), and a GFP filter set (49002, Chroma) was used. Images were acquired at $10 \mathrm{~Hz}(2 \mathrm{x}$

9262 digital binning, $1024 \times 1024$ pixel resolution) with an sCMOS camera (Prime BSI, Teledyne

927 Photometrics) using Micro-manager open-source software for $30 \mathrm{sec}$ before and $150 \mathrm{sec}$ after

928 taltirelin application. EZcalcium open-source MATLAB toolbox ${ }^{41}$ was used for motion

929 correction and automated ROI (region of interest) detection. Round-shaped, soma-like ROIs

930 were manually selected for extracting neuronal fluorescence signals. Fluorescence signal of each

931 MEA slice was obtained by averaging changes in fluorescence (deltaF/F) of individual neurons

932 ( $\mathrm{n}=336$ cells from 6 slices). The fluorescence signal of individual MEA slices were used to

933 obtain the average fluorescence trace shown in Extended Data Fig. 9k.

934

935 In vivo recording of MEA responses upon photoactivation of COApm inputs

936 MEA responses to COApm inputs in conditional Trhr KO mice were tested by recording local

937 field potentials (LFPs) in anesthetized mice. $\mathrm{Trhr}^{\mathrm{fl} / f l}$ mice were unilaterally injected with $\mathrm{AAV}_{2-}$

938 hSyn-ChR2-EYFP in COApm and $\mathrm{AAV}_{1}$-hSyn-GFP or $\mathrm{AAV}_{1}$-hSyn-Cre in the ipsilateral

939 MEApv. Four weeks after the viral injections, mice were anesthetized using a mixture of

940 ketamine $(100 \mathrm{mg} / \mathrm{kg}$, intraperitoneal injection) and xylazine $(10 \mathrm{mg} / \mathrm{kg}$, intraperitoneal 
941 injection) and placed in a stereotaxic frame. An homemade optrode, which consists of an optic

942 fiber (200um) and two-channel tungsten electrodes $(5 \mathrm{M} \Omega, 300 \mathrm{um}$ projection from the optic fiber

943 tip), were lowered to MEApv. LFPs were recorded starting at DV=-5.0 $\mathrm{mm}$ from the bregma,

944 while the electrode was being lowered, to find the locus of the maximum response evoked by a

945 single pulse of photoactivation (405nm laser, $2 \mathrm{~mW}, 10-\mathrm{msec}$ ) of COApm inputs. Recordings

946 were amplified, band-pass filtered between 10 and $300 \mathrm{~Hz}$ and digitized at $10 \mathrm{KHz}$ using a

947 Digital Lynx 4SX system (Neuralynx). TTL event signals for photoactivation from a pulse

948 generator were aligned to the recorded waveforms using the Neuralynx system. Light-evoked

949 local field potentials were analyzed off-line using a (ADInstrument, NeuroExplorer5). Three

950 waveforms evoked by photostimulations delivered at 1-min intervals were averaged to obtain the

951 baseline-to-negative peak amplitude for individual animals.

952

\section{RNA sequencing}

954 Labeling of COApm cells projecting to MEA. Vglut2-Cre mice received stereotaxic injections of

955 a virus mixture (AAV1-syn-FLEX-TTA and AAV1-TRE-B19G, total $0.5 \mu 1)^{42}$ into MEApv $( \pm 2.1$

956 ML, -1.7 AP, -5.6 DV). After 2 weeks, glycoprotein gene deleted rabies virus (RV $\Delta \mathrm{G}-4 \mathrm{EGFP}-$

957 L10a (EnvA)) was targeted to the same coordinates. Five days later, COApm tissues were

958 harvested and immediately used for TRAP purifications as described below. For the control 959 group, $100 \mu$ l of AAV.PHP.eB-Syn-L10a-EGFP $\left(1.13 \times 10^{11} \mathrm{VG} /\right.$ mouse concentration; SignaGen

960 Laboratories, Frederick, MD) was retro-orbitally injected. COApm tissue was harvested at 3-

961 weeks post-injection and used immediately for TRAP purifications.

962 TRAP purifications. Brain tissue dissections were performed on ice after cooling the head in

963 liquid nitrogen for $4 \mathrm{sec}$. After dissection, cell type-specific mRNAs were purified according to 
964 the established TRAP protocol ${ }^{43}$. COApm tissue was homogenized with a glass/Teflon power965 driven Potter-Elvehjem homogenizer in an ice-cold lysis buffer (150mM KCl, $10 \mathrm{mM}$ HEPES 966 [pH 7.4], $5 \mathrm{mM} \mathrm{MgCl}_{2}, 0.5 \mathrm{mM}$ dithiothreitol, $100 \mu \mathrm{g} / \mathrm{mL}$ cycloheximide, $20 \mathrm{U} / \mu \mathrm{L}$ SUPERaseIn 967 RNase Inhibitor, $40 \mathrm{U} / \mu \mathrm{L}$ RNasin Plus Rnase Inhibitor, and EDTA-free protease inhibitors). 968 Following homogenization, samples were centrifuged at $2,000 \mathrm{x} g$ at $4{ }^{\circ} \mathrm{C}$ for $10 \mathrm{~min}$ and the 969 supernatant was transferred to a new tube. NP-40 (final concentration 1\%) and 1,2-Diheptanoyl970 sn-glycero-3-phosphocholine (DHPC, final concentration $15 \mathrm{mM}$ ) were subsequently added and 971 samples were incubated on ice for $5 \mathrm{~min}$. Samples were centrifuged at $13,000 \mathrm{x} \mathrm{g}$ at $4^{\circ} \mathrm{C}$ for 10 972 min and the supernatant was transferred to a new tube. Streptavidin Dynabeads (ThermoFisher

973 Scientific, Waltham MA) coated with biotin-linked mouse anti-GFP antibodies were then added

974 to this supernatant and the samples were incubated overnight at $4{ }^{\circ} \mathrm{C}$ with end-over-end rotation.

975 Beads were collected on a magnetic rack and washed three times with wash buffer $(350 \mathrm{mM} \mathrm{KCl}$, 976 10mM HEPES [pH 7.4], $5 \mathrm{mM} \mathrm{MgCl}_{2}, 0.5 \mathrm{mM}$ dithiothreitol, $100 \mu \mathrm{g} / \mathrm{mL}$ cycloheximide, $1 \% \mathrm{NP}$ -

977 40). RNA was subsequently purified using the Absolutely RNA Isolation Nanoprep kit (Agilent

978 Technologies, Santa Clara CA). To ensure quality and accurate quantitation, purified RNA was 979 run on a Bioanalyzer using the RNA 6000 Pico Kit (Agilent Technologies, Santa Clara CA). 980 TRAP RNA sequencing and analysis. Purified TRAP samples were prepared for RNA-Seq using 981 the Takara Bio Smart-Seq v4 kit (Takara Bio USA, Inc.). RNA-Seq was performed on an 982 Illumina HiSeq 4000 at the University of California, San Francisco Functional Genomics Core.

983 FASTQ files were aligned to the Mus musculus genome build GRCm38 and annotation build 96

984 (reference FASTA and GTF file) using STAR v2.7.2b. Read counts were generated by STAR 985 using the --quantMode GeneCounts option, using the default options. Only mapped reads 986 uniquely assigned to the mouse genome were used for differential expression testing. These were 
987 imported into R and then converted to normalized read counts with DESeq2 ${ }^{44}$. Differential 988 Expression was performed using DESeq2, and significant genes were filtered by a q-value (False 989 Discovery Rate) threshold of 1e-15. To remove systemic bias possible from the use of rabies $990 v^{2 i r u s}{ }^{45,46}$, genes involved in the following MSigDB hallmark gene sets were further removed: 991 Interferon Gamma Response, Interferon Alpha Response, Allograft Rejection, IL6 JAK STAT3 992 Signaling, TNFa Signaling via NF-kB, Inflammatory Response, Complement, G2M Checkpoint, 993 E2F Targets, and IL2 STAT5 Signaling. These differentially expressed genes were then 994 compared to the set of COApm-enriched genes [relative to the grey matter] obtained from Allen 995 Brain Atlas Differential Search with the expression threshold set to 1. For KEGG pathway 996 analysis, differentially expressed genes were processed using Enrichr ${ }^{47,48}$.

\section{Statistics and reproducibility}

999 Statistical analyses were performed using GraphPad Prism. Sample sizes were chosen on the 1000 basis of similar previous studies ${ }^{20,49}$, and not on statistical methods to predetermine sample sizes.

1001 Within each iteration of an experiment, mice were randomly assigned to groups with 1002 approximately balanced sample size. Behavioral results from mice with inaccurate targeting of 1003 viral infection or cannula implantations were excluded.

1004

1005 Data availability

1006 Source data are provided in the supplementary information. Sequencing datasets are publicly 1007 available in NCBI GEO under accession \# GSE167176. All data are available from the 1008 corresponding author upon request. 